\title{
PROPUESTA DE PERFECCIONAMIENTO PARA LA FORMACIÓN DEL PENSAMIENTO REFLEXIVO DESDE EL PROCESO DE ENSEÑANZA APRENDIZAJE DE LA MATEMÁTICA EN CARRERAS DE INGENIERÍA
}

\author{
María Teresa Ruiz García ${ }^{1}$ \\ https://orcid.org/0000-0002-1643-1230 \\ Raquel Diéguez Batista ${ }^{2}$ \\ https://orcid.org/0000-0002-4975-6947 \\ Raudel Torrecilla Díaz ${ }^{3}$ \\ https://orcid.org/0000-0002-6106-1953
}

\begin{abstract}
RESUMEN
Este artículo se realiza en base a los impedimentos que presentan los estudiantes que cursan las carreras de Ingeniería en la Universidad de Ciego de Ávila Máximo Gómez Báez, referente a la solución de problemas matemáticos con apoyo heurístico; tendencia a trabajar por patrones en su solución y dificultades en los niveles de argumentación para valorar los resultados. El objetivo que se persigue es la elaboración de un Sistema de procedimientos didácticos para la formación del pensamiento reflexivo desde la enseñanza aprendizaje de la Matemática en las carreras de Ingeniería, sustentado en un Modelo del proceso de formación del pensamiento reflexivo desde este proceso formativo, usando como principal referente a: Moreno (2011). En la modelación se aportan relaciones dialécticas que fundamentan la necesidad del acondicionamiento de las estructuras cognitivas para la apropiación del nuevo contenido, su reconstrucción progresiva y establecimiento, para la solución de problemas matemáticos ingenieriles con un razonamiento lógico, lo que posibilita transitar de forma gradual por diferentes niveles de sistematización de contenidos y de apropiación de procedimientos lógicos reflexivos. El método holístico dialéctico se utilizó en la construcción del Modelo de la dinámica de formación del pensamiento reflexivo en el proceso de enseñanza aprendizaje de la Matemática en las carreras de Ingeniería. Al confeccionar el Sistema de procedimientos didácticos, se utilizó el método sistémico estructural; donde se establecen acciones para guiar el proceso de formación del pensamiento reflexivo. El enfoque hermenéutico dialéctico, propició la interpretación de los datos tendenciales, empíricos y teóricos, partiendo de su comprensión y explicación, por lo que la investigación transitó por diferentes niveles de sistematización epistemológica y metodológica. Los resultados obtenidos se someten a Criterio de Expertos, estos coinciden en la adecuación científica metodológica de la investigación, por lo que su aplicación parcial corrobora el valor práctico de los resultados.

Palabras Claves: Pensamiento Reflexivo; Sistema de Procedimientos; Ingeniería; Resolución de Problemas matemáticos; Formación de profesionales.
\end{abstract}

Proposta de melhoria para a formação do pensamento refletivo a partir do processo de ensino aprendizagem da matemática na carreira de engenharia.

\section{RESUMO}

Este artigo baseia-se nas limitações apresentadas por estudantes do curso de Engenharia da Universidade de Ciego de Ávila Máximo Gómez Báez, referentes à solução de problemas matemáticos com suporte heurístico; tendência a trabalhar por padrões em sua solução e dificuldades nos níveis de argumentação para avaliar os resultados. O objectivo prosseguido é o desenvolvimento de um sistema de procedimentos didácticos para a formação do pensamento reflexivo a partir do ensino-aprendizagem da Matemática nas carreiras da Engenharia, com base em um Modelo do processo de formação do pensamento reflexivo a partir desse processo formativo, tendo como principal referência, Moreno (2011). Na modelagem foram previstas relações dialécticas que sustentam a necessidade de condicionamento de estruturas cognitivas para a apropriação de novos conteúdos, sua progressiva reconstrução e estabelecimento, para a solução de problemas matemáticos relacionados com a engenharia, utilizando raciocínio lógico, o que o torna

\footnotetext{
${ }^{1}$ Universidad Máximo Gómez Báez de Ciego de Ávila, Cuba. Email: mariateresa@unica.cu

2 Universidad Máximo Gómez Báez de Ciego de Ávila, Cuba. Email: raquel@unica.cu

${ }^{3}$ Universidad Máximo Gómez Báez de Ciego de Ávila, Cuba.
} 
possível passar gradativamente por diferentes níveis de sistematização de conteúdo e apropriação de procedimentos lógicos reflexivos. O Método holístico - dialéctico foi utilizado na construção do modelo da dinâmica de formação do pensamento reflexivo no processo ensino-aprendizagem da Matemática nas carreiras de Engenharia. Na elaboração do sistema de procedimentos didácticos, foi utilizado o método estrutural sistémico, onde se estabelecem acções para orientar o processo de formação do pensamento reflexivo. A abordagem hermenêutica dialéctica conduziu à interpretação da tendência, dados empíricos e teóricos, a partir de sua compreensão e explicação, para os quais a pesquisa passou por diferentes níveis de sistematização epistemológica e metodológica. Os resultados obtidos foram submetidos a Critérios de Especialistas que, coincidem na adequação metodológica científica da pesquisa e a sua aplicação parcial corrobora o valor prático dos resultados.

Palavras-chave: Pensamento reflexivo; Sistema de procedimentos; Engenharia; Resolução de problemas matemáticos; Formação de profissionais.

Improvement proposal for the training of reflective thinking from the process of teaching learning of mathematics in engineering careers.

\section{ABSTRACT}

This article is based on the impediments presented by students studying Engineering at the University of Ciego de Ávila Máximo Gómez Báez, referring to the solution of mathematical problems with heuristic support; tendency to work by patterns in their solution and difficulties in the levels of argumentation to assess the results. The objective pursued is the development of a System of didactic procedures for the formation of reflective thinking from the teaching-learning of Mathematics in Engineering careers, supported by a Model of the training process of reflective thinking from this formative process, using as main reference, Moreno (2011). In the modeling dialectical relationships are provided that support the need for conditioning of cognitive structures for the appropriation of new content, its progressive reconstruction and establishment, for the solution of mathematical engineering problems with reasoning logical, which makes it possible to gradually go through different levels of content systematization and appropriation of reflective logical procedures. The holistic-dialectical method was used in the construction of the Model of the training dynamics of reflective thinking in the teaching-learning process of Mathematics in Engineering careers. When making the System of didactic procedures, the structural systemic method was used, where actions are established to guide the process of formation of reflective thought. The dialectical hermeneutical approach, led to the interpretation of the trend, empirical and theoretical data, starting from its understanding and explanation, for which the research went through different levels of epistemological and methodological systematization. The results obtained are submitted to Expert Criteria, these coincide in the scientific methodological adequacy of the research, so their partial application corroborates the practical value of the results.

Keywords: Reflective Thinking; Procedures System; Engineering; Solving Mathematical Problems; Training of professionals.

\section{Introducción}

La formación de ingenieros capacitados para enfrentar los retos de este siglo, constituye un desafío para las universidades. Actualmente las universidades demandan una formación integral en los egresados, donde el proceso de enseñanza aprendizaje debe estar encaminado a potenciar el desarrollo académico integral de ingenieros que responda a las exigencias de la sociedad contemporánea. Sin embargo, a pesar de los esfuerzos realizados se observa una contradicción en la práctica, entre las exigencias formativas reconocidas en el modelo del profesional de las carreras de Ingeniería y las limitaciones que se observan en la aplicación de las herramientas de la Matemática, así como en el razonamiento lógico matemático requerido en el planteamiento y solución de problemas. Esta situación es reconocida por autores como Paulino (2019) y Camoli, Ferreira, Pino (2020), quienes afirman que las anteriores consideraciones son las que predominan en los estudiantes de nivel universitario y a la vez consideran que esta ciencia es la base de conocimientos con mayor importancia para la sociedad contemporánea. Moreno (2011) y Castro (2020), identifican que existen varios factores que la originan entre los que se encuentra: la población generalmente siente rechazo por la Matemática, una enseñanza inadecuada, existen pocos materiales y recursos didácticos para el proceso enseñanza aprendizaje de esta ciencia, así como insuficiencia en la formación didáctico metodológica de los profesores. Se coincide con Moreno (2011), cuando refiere que las dificultades en el aprendizaje de la Matemática deben constituir un objeto de preocupación de los profesores; dándole solución a las diferentes 
dificultades, a medida que los estudiantes adquieren conocimientos de esta ciencia, que se considerada tradicionalmente difícil de entender. Por otra parte en el modelo del profesional se establece que la disciplina Matemática se encuentra dentro del currículo de formación del Ingeniero, esta disciplina es una herramienta importante para darle solución a los problemas; donde los estudiantes apliquen conocimientos y procedimientos para la comprensión de los procesos ingenieriles. Se revela entonces una contradicción entre la preparación de los estudiantes en la solución a problemas matemáticos con un razonamiento lógico y las exigencias formativas reconocidas en el modelo del profesional de las carreras de Ingeniería.

Teniendo en cuenta estos elementos se realizaron diagnósticos a los 12 estudiantes de primer año de la carrera Ingeniería Hidráulica y a 9 profesores del Departamento de Matemática de la Universidad de Ciego de Ávila Máximo Gómez Báez, curso 2018-2019; fundamentado en análisis documental de controles a clases, encuestas a profesores y prueba diagnóstico. Se concretan las siguientes manifestaciones en los estudiantes: dificultades al utilizar los conocimientos matemáticos en la solución de problemas con apoyo heurístico; tendencia a trabajar por patrones en la solución de problemas matemáticos, que limitan la búsqueda de alternativas novedosas y efectivas; dificultades en los niveles de argumentación para valorar los resultados de los problemas matemáticos. Estas manifestaciones se concretan en el Problema de investigación: Insuficiencias en la solución de problemas matemáticos, en relación con el razonamiento lógico requerido en la Matemática. Al profundizar en el estudio realizado, se revela la siguiente causa del problema enunciado con anterioridad: inconsistencias en los posicionamientos epistemológicos asumidos en el proceso enseñanza aprendizaje de la Matemática en las carreras de Ingeniería, con sesgos en su direccionamiento hacia la construcción de estructuras lógicas de pensamiento y la relación ciencia profesión, como condiciones necesarias para la apropiación de contenidos. El objeto de estudio de la presente investigación es el proceso de enseñanza aprendizaje de la Matemática en las carreras de Ingeniería.

En el estudio del objeto se revela un insuficiente soporte metodológico para la formación del pensamiento reflexivo en la asignatura Matemática. Por lo que es pertinente la elaboración de un Sistema de procedimientos didácticos del proceso de enseñanza aprendizaje de la Matemática en las carreras de Ingeniería, sustentado en un Modelo del proceso de formación del pensamiento reflexivo desde el proceso de enseñanza aprendizaje de la Matemática. El campo de acción es la formación del pensamiento reflexivo en el proceso de enseñanza aprendizaje de la Matemática en las carreras de Ingeniería. En la fundamentación epistemológica y metodológica del objeto y el campo de acción de esta investigación, se logra configurar la necesidad de potenciar, desde el proceso de sistematización de contenidos matemáticos, la apropiación de procedimientos lógicos en el ingeniero en formación, lo que posibilita la solución de problemas matemáticos ingenieriles, con un pensamiento reflexivo, por lo que se plantea como hipótesis: Si se elabora un Sistema de procedimientos didácticos para el proceso de enseñanza aprendizaje de la Matemática en las carreras de Ingeniería, que tenga en cuenta la relación entre la sistematización de contenidos y la apropiación de procedimientos lógicos reflexivos, se puede contribuir a atenuar las insuficiencias que presentan los estudiantes de estas carreras en la solución de problemas matemáticos, en relación con el razonamiento lógico requerido en esta ciencia.

Se aplicaron los siguientes métodos y técnicas: el método histórico lógico, se utilizó fundamentalmente para la caracterización de las tendencias históricas para la formación del pensamiento reflexivo en el proceso de enseñanza aprendizaje de la Matemática en las carreras Ingeniería y la formación del pensamiento reflexivo desde este proceso formativo; los métodos de análisis síntesis y abstracción concreción, transitaron por el proceso de la investigación científica, fundamentalmente en la caracterización del objeto y campo de acción del trabajo; el método holístico-dialéctico: se utilizó en la construcción del Modelo de la dinámica de formación del pensamiento reflexivo en el proceso de enseñanza aprendizaje de la Matemática en las carreras de Ingeniería; el método sistémico estructural funcional fundamentalmente en la elaboración del 
Sistema de procedimientos didácticos; el criterio de experto para valorar la pertinencia científico metodológica de los resultados, lo cual se argumenta posteriormente en la Valoración de la pertinencia del Sistema de procedimientos didácticos mediante Criterio de Expertos.

Métodos Estadísticos: Estadística Descriptiva para el análisis de los resultados del diagnóstico y de la aplicación parcial del Sistema de procedimientos didácticos propuesto; estadística Inferencial: pruebas de hipótesis no paramétrica para corroborar las transformaciones existentes al aplicarse el Sistema de procedimientos didácticos propuesto; encuesta, prueba de conocimiento y análisis documental para corroborar las manifestaciones y causas del problema, así como en la caracterización del estado actual del campo de acción en la Universidad de Ciego de Ávila.

La deducción de los datos tendenciales, empíricos y teóricos, desde su aprehensión y descripción se interpretaron fácilmente, mediante el enfoque hermenéutico dialéctico, como consecuencia de los niveles de sistematización metodológicos e epistemológicos que transitó la investigación, materializándose en el proceso interpretativo.

La contribución a la teoría es un Modelo de la dinámica de formación del pensamiento reflexivo en el proceso de enseñanza aprendizaje de la Matemática en las carreras de Ingeniería. El aporte práctico es el Sistema de procedimientos didácticos para la formación del pensamiento reflexivo en el proceso de enseñanza aprendizaje de la Matemática en carreras de Ingeniería. La significación práctica de la investigación se evidencia en el actuar de los estudiantes que cursan las carreras de Ingeniería de la Universidad Máximo Gómez Báez, al enfrentar la solución de problemas matemáticos ingenieriles con apoyo heurístico; búsqueda de diferentes alternativas novedosas y efectivas; así como una argumentación concisa en la valoración de los resultados. La novedad científica de la investigación se expresa en las transformaciones que se revelan en el proceso de formación del pensamiento reflexivo en la enseñanza aprendizaje de la Matemática en carreras de Ingeniería desde la preparación de las estructuras cognitivas para la apropiación del nuevo contenido, su reconstrucción progresiva donde se afiancen y reafirmen los conocimientos anteriores y posteriores y el establecimiento de las estructuras cognitivas, que posibilitan transitar por diferentes niveles de sistematización de contenidos y apropiación de procedimientos lógicos reflexivos.

\section{Fundamentación epistemológica de la formación del pensamiento reflexivo en el proceso de enseñanza aprendizaje de la Matemática en las carreras de Ingeniería}

Se analizan los postulados de Ruiz (2006), Barros (2010), Ritacco (2012), Germán (2017), la contextualización del contenido es esencial implementarla en situaciones de la Matemática en la práctica social. La idea fundamental es que en la solución de problemas el estudiante llegue a la comprensión, creatividad, imaginación, racionalidad secuencial, así como a la selección y utilización de métodos para resolverlos, desde procesos cognitivos que conllevan a un pensamiento lógico. Los presupuestos epistemológicos de la lógica dialéctica planteada por: Lenin (1964), Lienkov (1977) y Davydov (1986), manifiestan que mediante la práctica, el pensamiento proporciona resultados en el proceso de formación de conceptos; esto es fundamental dentro del proceso de enseñanza aprendizaje de la Matemática, específicamente en la solución de problemas matemáticos, pues se aplican conceptos, se realizan juicios, mediante un razonamiento lógico, llegando a tener una nueva concepción de la solución del problema. Según lo planteado por Faustino (2014), el desarrollo de formas del pensamiento lógico (ideas, juicios y razonamientos), conduce a la identificación de conceptos matemáticos y determina las actividades mentales en dependencia de las condiciones en que se produce el movimiento de un juicio a otro, en una dialéctica, a partir de la apropiación de un sistema de procedimientos lógicos. Desarrollar un pensamiento lógico, implica el desarrollo de actividades secuenciadas y relacionadas hasta llegar a dar respuesta coherente a una situación problémica planteada, donde el estudiante en la solución de problemas llegue a la comprensión, creatividad, imaginación, racionalidad secuencial, así como 
a la selección y utilización de métodos para resolverlo, desde procesos cognitivos que conllevan a un pensamiento lógico.

En relación con el pensamiento lógico está el pensamiento reflexivo. Halpern (1998), Elder y Paul (2010), Ennis (2013), relacionan que en el pensamiento reflexivo intervienen las disposiciones y habilidades que tengan las personas para hacer algo, estas dos cualidades son de vital importancia, pues una persona que no tenga interés para realizar una tarea no la ejecuta adecuadamente, ni toma tiempo para reflexionar en ella, no piensa de forma reflexiva, por lo que hay que tener en cuenta estas dos cualidades para la formación del pensamiento reflexivo en los estudiantes. Según la teoría de Duval (1993), el pensamiento reflexivo en el primer nivel se centra en observar, analizar y proponer. En un segundo nivel podrá reflexionar al resolver actividades en el aula y argumentar sus respuestas de forma escrita y oral. En un tercer nivel aplicará el razonamiento lógico en la solución de problemas, integrar informes escritos y exposiciones orales con argumentos bien sustentados. El pensamiento reflexivo está estatuido por acciones, estas acciones interactúan unas con otra, dependiendo una de la otra, dando lugar a una base cognitiva que crea estructuras lógicas conscientes en el hombre, estas estructuras son las responsables de construir y modificar el pensamiento, evidenciándose que la formación del pensamiento reflexivo en nuestra sociedad ocupa un lugar primordial, una persona con pensamiento reflexivo tiene la capacidad de realizar actos deductivos, demostraciones y conclusiones lógicas aplicables, ampliando en gran grado las posibilidades del conocimiento

Las anteriores consideraciones permitieron definir el pensamiento reflexivo como la capacidad de emitir un criterio en la solución de un problema, utilizando ideas preconcebidas que se basan en la relación entre los conceptos ya conocidos y los que se perciben, donde intervienen la memoria, comprensión y aprendizaje, llegando a la formación de nuevos criterios.

Estas investigaciones revelan la importancia que tiene, en el proceso de enseñanza aprendizaje de la Matemática la formación del pensamiento reflexivo; para la comprensión del rol de esta ciencia, así como la organización e interpretación de la información, el desarrollo de la creatividad e interés por el descubrimiento y las habilidades para analizar e interpretar problemas mediante la comunicación de métodos y la justificación de procesos.

En la presente investigación se clasifican los problemas matemáticos en: preparatorios, integradores e ingenieriles. Los primeros se refieren a aquellos relacionados con los contenidos matemáticos que constituyen prerrequisito de la disciplina; en los integradores se relaciona lo conocido y lo buscado progresivamente, tratando de encontrar los elementos cognitivos que permiten resolverlo mediante el razonamiento lógico que conlleve a la estructuración y organización de la información que proporciona la realidad. Los ingenieriles son problemas del contexto profesional que se resuelven mediante métodos matemáticos (Torrecilla, 2015). Se definen los procedimientos lógicos reflexivos como un conjunto estructurado de acciones lógicas del intelecto, previamente meditadas que cumplen un objetivo determinado, esta definición está sustentada por los planteamientos de: Ackoff (1986), que define el procedimiento reflexivo como aquel donde se toman ideas de los sentidos, se pasan al intelecto dándole forma, estructura lógica y contenido, facilitando de esa manera la internalización de la realidad, y de Oviedo (2015), que define los procedimientos lógicos como el sistema de acciones basadas en el empleo adecuado del análisis, la síntesis, la abstracción y la generalización que permiten obtener un reflejo correcto de la realidad.

Por otro lado, Fuentes (2009), define la sistematización del contenido como el proceso mediante el cual la persona es capaz de generalizar sus habilidades y sus conocimientos con el fin de obtener un objetivo específico, como fruto de la relación dialéctica entre el nivel de las potencialidades intelectuales y el nivel de profundidad del contenido, que se pone de manifiesto en el objeto de estudio. Se requiere llevar al proceso de formación matemática para que el estudiante pueda enfrentar el proceso de solución de problemas, pero integrada a la sistematización de métodos que viabilicen la concreción de procedimientos algebraicos desde la indagación (Faustino, 2014). Por 
lo cual, se debe abordar el proceso de enseñanza aprendizaje de la matemática desde la sistematización del contenido y la contextualización interdisciplinar, mediante fundamentos didácticos y psicológicos, sustentado en los procedimientos lógicos reflexivos que resulten viables para la formación del pensamiento reflexivo de los estudiantes en la preparación profesional, desde una didáctica que justifique la integración de los niveles interpretativos y la secuencia del proceso de solución de problemas matemáticos ingenieriles, propiciando cambios dinámicos en la formación del pensamiento mediante la formulación de leyes, problemas, definiciones e hipótesis, que superen obstáculos epistemológicos del pensamiento reflexivo de los estudiantes.

Por su parte Campistrous (1992), define tres formas lógicas en que se manifiesta el pensamiento, como son: conceptos, juicios y razonamientos, asociadas por procedimientos lógicos. Tanto los procedimientos como las formas lógicas se retoman en esta investigación, donde se tiene en cuenta principalmente los procedimientos lógicos.

Tendencias históricas para la formación del pensamiento reflexivo en el proceso de enseñanza aprendizaje de la Matemática en las carreras de Ingeniería

Se inicia el estudio de las tendencias históricas para la formación del pensamiento reflexivo en el proceso enseñanza aprendizaje de la Matemática en carreras de Ingeniería, apoyándose en los siguientes indicadores de análisis: métodos que prevalecen en el proceso de enseñanza aprendizaje de la Matemática; caminos utilizados para la formación del pensamiento reflexivo en la enseñanza aprendizaje de la Matemática; relación ciencia profesión en el proceso enseñanza aprendizaje de la Matemática.

En correspondencia con los cambios ocurridos en el período analizado, desde el análisis de los indicadores establecidos, se definen las siguientes etapas:

Etapa 1. Proceso de enseñanza aprendizaje de la Matemática descontextualizado y memorístico que transita desde 1962 hasta 1979 (Ministerio de Educación Superior, 1977)

Etapa 2. Reconocimiento de la solución de problemas como centro de la enseñanza aprendizaje de la Matemática que transita desde 1980 hasta 2002 (Hernández, 1995); (Ministerio de Educación Superior, 1986); (Ministerio de Educación Superior, 1990)

Etapa 3. Sistematización de la solución de problemas matemáticos aplicados al perfil profesional que transita desde 2003 hasta el presente (Ministerio de Educación Superior, 2003); (Ministerio de Educación Superior, 2018)

En general, el análisis de los indicadores por etapas reveló que el proceso de enseñanza aprendizaje de la Matemática en carreras de Ingeniería ha transitado desde métodos básicamente reproductivos, así como la solución algorítmica de problemas, con limitada interpretación del proceso y de resultados, hacia la formación problematizada que integra situaciones problémicas y problemas matemáticos ingenieriles, sin embargo todavía no se logra desarrollar la independencia cognoscitiva y el pensamiento reflexivo de los estudiantes en la solución de problemas vinculados a la profesión.

Caracterización del estado actual de la formación del pensamiento reflexivo desde el proceso de enseñanza aprendizaje de la Matemática en las carreras de Ingeniería

Para la caracterización del estado actual de la formación del pensamiento reflexivo desde el proceso de enseñanza aprendizaje de la Matemática en las carreras de Ingeniería de la Universidad de Ciego de Ávila Máximo Gómez Báez, se procedió inicialmente al análisis documental de los planes de estudio (Recuadro 1) de estas carreras de Ingeniería (Ministerio de Educación Superior, 2018), con el propósito de corroborar las exigencias, que desde estos documentos se establecen para la formación del pensamiento reflexivo de los estudiantes en la enseñanza aprendizaje de la Matemática, así como la intencionalidad de establecimiento de la relación ciencia-profesión en este proceso formativo, cuestiones evidenciadas desde la fundamentación de la disciplina y los objetivos. Posteriormente se corrobora el problema de investigación y se caracteriza el estado 
actual del proceso de formación del pensamiento reflexivo en las carreras de Ingeniería en la Universidad de Ciego de Ávila Máximo Gómez Báez, mediante la aplicación de diferentes instrumentos: Prueba de Rigidez del Pensamiento de Davydov (Tabla 1) y la Prueba de conocimiento que se les realizó a 12 estudiantes de primer año de la carrera Ingeniería Hidráulica, curso 2018-2019 (Recuadro 2); encuestas a 9 profesores del colectivo de la disciplina Matemática, curso 2018-2019 (Recuadro 3) y revisión de 8 controles a clases que les realizó a los profesores de la disciplina, curso 2018-2019 (Recuadro 4).

Recuadro 1. Guía de análisis documental de los planes de estudio de las carreras de Ingeniería de la Universidad de Ciego de Ávila Máximo Gómez Báez.

Documento: Planes de estudio de las carreras de Ingeniería de la Universidad de ciego de Ávila Máximo Gómez Báez.

Objetivo: Determinar las exigencias para la formación del pensamiento reflexivo en el proceso de enseñanza aprendizaje de la Matemática en las carreras de Ingeniería.

Universo de estudio: Plan de Estudio de las carreras de Ingeniería de la Universidad de

Ciego de Ávila Máximo Gómez Báez. (Ministerio de Educación Superior, 2018)

Muestra de aspectos a analizar: Modelo del profesional y programas de disciplinas.

Categorías de análisis:

- Exigencias manifiestas para el desarrollo del pensamiento reflexivo en la enseñanza aprendizaje de la Matemática.

- Intencionalidad de establecimiento de la relación ciencia-profesión en el proceso enseñanza aprendizaje de la Matemática.

Codificador: Investigador

Fuente: Elaboración propia

Tabla 1. Prueba de Rigidez del Pensamiento Davydov que se realizó a 12 estudiantes de primer año de la carrera de Ingeniería Hidráulica de la Universidad de Ciego de Ávila Máximo Gómez Báez en el curso 2018-2019

Objetivo: Determinar las especificaciones de la manifestación de la rigidez del pensamiento en los estudiantes durante la resolución de cada tarea aritmética propuesta.

Metodología para la realización de la prueba: Las tareas deben realizarse de forma escrita en no más de dos minutos. Al resolver la tarea $\mathrm{No}(0)$ las restantes se realizan cada dos minutos.

\begin{tabular}{|l|l|l|l|l|}
\hline $\begin{array}{l}\text { No de } \\
\text { la Tarea }\end{array}$ & \multicolumn{3}{|l|}{ Volumen de los } & $\begin{array}{l}\text { Cantidad } \\
\text { necesaria } \\
\text { de líquido }\end{array}$ \\
\hline o en litros & - & 29 & 3 & 20 \\
\hline 1 & 14 & 59 & 10 & 25 \\
\hline 2 & 14 & 163 & 25 & 99 \\
\hline 3 & 28 & 43 & 10 & 5 \\
\hline 4 & 31 & 61 & 4 & 22 \\
\hline 5 & 18 & 59 & 7 & 27 \\
\hline 6 & 23 & 49 & 3 & 20 \\
\hline 7 & 15 & 39 & 3 & 18 \\
\hline 8 & 28 & 76 & 3 & 25 \\
\hline 9 & 28 & 48 & 4 & 12 \\
\hline 10 & 14 & 36 & 8 & 6 \\
\hline
\end{tabular}

Fuente: Davydov (1986). 
Recuadro 2. Prueba de conocimiento que se realizó a 12 estudiantes de primer año de la carrera de Ingeniería Hidráulica, de la Universidad de Ciego de Ávila Máximo Gómez Báez en el curso 2018-2019.

Objetivo: Determinar las insuficiencias que presentan los estudiantes de la carrera de Ingeniería Hidráulica en la solución de problemas matemáticos ingenieriles, en relación con el razonamiento lógico requerido en la Matemática.

Un recipiente de agua tiene forma cilíndrica con una altura de $10 \mathrm{~m}$, el perímetro de la circunferencias base es de $5 \mathrm{~m}$ y el área total del recipiente es de $650 \mathrm{~m}^{2}$

a) Determina la cantidad máxima de agua del recipiente.

b) ¿Cuántas horas demorará en vaciarse cuando está lleno al $60 \%$ si por la llave de salida se extraen 55 litros por minuto?

c) Si se desea pintar toda la superficie del reciente y sabe para ello que la cantidad de pintura que se utiliza en una de sus bases es 2 litros. ¿Cuál será la cantidad de pintura a utilizar?

Fuente: Elaboración propia

Recuadro 3. Encuesta que se realizó a 9 profesores de la disciplina Matemática para las carreras de ingeniería de la Universidad de Ciego de Ávila Máximo Gómez Báez en el curso 2018-2019.

Objetivo: Caracterizar el proceso de formación del pensamiento reflexivo en el proceso de enseñanza aprendizaje de la Matemática en las carreras de Ingeniería. Instructivo: Usted debe evaluar en una escala del 1 al 5 cada uno de los aspectos que se señalan.

5- excelencia4- muy bien3- bien2- regular 1- mal

1. Durante el desarrollo de una clase la mayor parte del tiempo los estudiantes exponen sus criterios.

2. ¿Crea con frecuencia las condiciones para que sus estudiantes tengan experiencias directas relacionadas con los temas a trabajar?

3. ¿Respondería preguntas en clases que no tienen que ver con el tema que se está tratando de resolver pero que son importantes para el estudiante que las realiza?

4. ¿En las diferentes actividades docentes plantea a los estudiantes problemas contextualizados a la realidad ingenieril?

5. ¿Debate con los estudiantes la selección de las alternativas de solución de ejercicios y problemas y su relación con la realidad ingenieril?

6. ¿Exige en sus clases la interpretación de las soluciones matemáticas de los problemas ingenieriles que se resuelven en correspondencia con la aplicabilidad del resultado?

7. ¿Considera adecuado orientar tareas extraclases que requieran que el estudiante visite empresas y otras entidades?

8. ¿Se orientan tareas a los estudiantes que requieran de la búsqueda de contenido no tratado en clases?

9. ¿Contribuye a la formación del pensamiento reflexivo en sus estudiantes desde el proceso de enseñanza aprendizaje de la Matemática?

10. Al abordar un tema permite que la imaginación de sus alumnos los lleve a ubicar los aspectos relevantes.

11. Al abordar un tema presenta todos los aspectos que considera relevante.

12. Al abordar un tema presenta una parte deja que sus alumnos encuentren el resto a través de lecturas, ejercicios, conversación heurística.

Fuente: Elaboración propia 
Recuadro 4. Guía de análisis documental de 8 controles a clases de Matemática que se realizaron en el departamento de Matemática de la Universidad de Ciego de Ávila Máximo Gómez Báez en el primer semestre del curso 2018-2019.

Objetivo: Caracterizar la formación del pensamiento reflexivo en el proceso de enseñanza aprendizaje de la Matemática en las carreras de Ingeniería de la Universidad de Ciego de Ávila Máximo Gómez Báez.

Universo de estudio: La totalidad de los controles a clases de Matemática realizados en el departamento de Matemática de la Universidad de Ciego de Ávila Máximo Gómez Báez en el primer semestre del curso 2018.

Muestra de aspectos a analizar: Métodos utilizados.

Categorías de análisis:

- Métodos que prevalecen en el proceso de enseñanza aprendizaje de la Matemática.

- Vías utilizadas para el desarrollo del pensamiento reflexivo en la enseñanza aprendizaje de la Matemática.

- Relación que se establece ciencia-profesión en el proceso enseñanza aprendizaje de la Matemática.

Fuente: Elaboración propia

Para este diagnóstico, se selecciona una muestra intencional de 12 estudiantes de la carrera Ingeniería Hidráulica del Curso Regular Diurno 2018-2019 y 9 profesores del departamento de Matemática de la Universidad de Ciego de Ávila Máximo Gómez Báez.

$\mathrm{Al}$ aplicar la prueba de conocimiento (Recuadro 2), en el año 2018, se observa que de 12 estudiantes presentados, el $83.4 \%$ presenta problemas para analizar, sintetizar, argumentar, generalizar y valorar, expresados en la precisión del método de solución, razonamiento, su aplicación y valoración de los resultados (Tabla 2).

Tabla 2. Resultados obtenidos de la Prueba de Conocimiento, que se realizó a 12 estudiantes de primer año de la carrera de Ingeniería Hidráulica de la Universidad de Ciego de Ávila Máximo

Gómez Báez en el curso 2018-2019.

Fuente: Elaboración propia

\begin{tabular}{|ll|l|l|l|l|}
\hline & Frecuencia & Porcentaje & $\begin{array}{l}\text { Porcentaje } \\
\text { válido }\end{array}$ & $\begin{array}{l}\text { Porcentaje } \\
\text { acumulado }\end{array}$ \\
\hline \multirow{4}{*}{ Válidos } & Muy Bajo & 8 & 66,7 & 66,7 & 66,7 \\
& Bajo & 2 & 16,7 & 16,7 & 83,4 \\
& Medio & 2 & 16,7 & 16,7 & 100,0 \\
& Total & 12 & 100,0 & 100,0 & \\
\hline
\end{tabular}

Se consideró oportuno aplicar a esta muestra de estudiantes, la Prueba de Rigidez del Pensamiento de Davydov (Tabla 1), la cual reveló que de los 12 estudiantes examinados, el 83,3\% tiene un pensamiento rígido, ante una situación nueva, es decir, tendencia a trabajar por patrones (Tabla 3).

Tabla 3. Resultados obtenidos de la Prueba de Rigidez del Pensamiento de Davydov, que se realizó a 12 estudiantes de primer año de la carrera de Ingeniería Hidráulica de la Universidad de Ciego de Ávila Máximo Gómez Báez en el curso 2018-2019.

\begin{tabular}{|ll|l|l|l|l|}
\hline & Frecuencia & Porcentaje & $\begin{array}{l}\text { Porcentaje } \\
\text { válido }\end{array}$ & $\begin{array}{l}\text { Porcentaje } \\
\text { acumulado }\end{array}$ \\
\hline \multirow{2}{*}{ Válidos } & Muy Bajo & 9 & 75,0 & 75,0 & 75,0 \\
& Bajo & 1 & 8,3 & 8,3 & 83,3 \\
& Medio & 2 & 16,7 & 16,7 & 100,0
\end{tabular}




\section{\begin{tabular}{|l|l|l|l|l|}
\hline Total & 12 & 100,0 & 100,0 & \\
\hline
\end{tabular}}

Fuente: Elaboración propia

Para conocer sus opiniones, a cerca de las dificultades que presentan los estudiantes y sus posibles causas, se realizó una encuesta a 9 profesores de la disciplina Matemática de la Universidad de Ciego de Ávila Máximo Gómez Báez en el curso 2018-2019 (Recuadro 3), los resultados obtenidos se relacionan a continuación: el $77.78 \%$ plantea que durante el desarrollo de una clase la mayor parte del tiempo los estudiantes exponen sus criterios, el $100 \%$ crean con frecuencia las condiciones para que sus estudiantes tengan experiencias directas relacionadas con los temas a trabajar; el 55.56\% plantea que expondría preguntas en clases que no tienen que ver con el tema que se está tratando de resolver pero que son importantes para el estudiante que las realiza; el $100 \%$ plantea que en las diferentes actividades, propone a los estudiantes problemas contextualizados a la realidad ingenieril; el 55.56\% debate con los estudiantes la selección de las alternativas de solución de ejercicios y problemas y su relación con la realidad ingenieril; el 88.89\% exige en sus clases la interpretación de las soluciones matemáticas de los problemas ingenieriles que se resuelven en correspondencia con la aplicabilidad del resultado; el 100\% considera adecuado orientar tareas extraclases que requieran que el estudiante visite empresas y otras entidades; el $33.3 \%$ plantea que orienta tareas a los estudiantes que requieran de la búsqueda de contenido no tratado en clases; el $66.67 \%$ plantea que contribuye a la formación del pensamiento reflexivo en sus estudiantes desde el proceso de enseñanza aprendizaje de la Matemática; el 55.56\% plantea que al abordar un tema deja que la imaginación de sus alumnos los lleve a ubicar los aspectos relevantes; el $77.78 \%$ plantea que al abordar un tema presenta todos los aspectos que considera relevante; el $33.33 \%$ plantea que al abordar un tema presenta una parte deja que sus alumnos encuentren el resto a través de lecturas, ejercicios, conversación heurística; el 100\% plantea que la tipología de clase utilizada en el proceso de enseñanza aprendizaje de la Matemática es la clase práctica.

Se procedió a la revisión de los controles 8 a clases de Matemática que se realizaron en el departamento de Matemática de la Universidad de Ciego de Ávila Máximo Gómez Báez en el primer semestre del curso 2018-2019 (Recuadro 4), con el propósito de determinar posibles causas de las insuficiencias presentadas, lo que posibilitó constatar que: ha existido un incremento en la utilización de métodos de enseñanza con un enfoque renovador y en función del tratamiento y solución de los problemas matemáticos ingenieriles de las diferentes carreras de Ingeniería, donde el pensamiento reflexivo constituye una herramienta imprescindible; aunque no se logra la trascendencia de la formación heurística e interpretativa para el ejercicio de la profesión con un pensamiento reflexivo; se evidencia un incremento en la realización de problemas matemáticos vinculados a la profesión; sin embargo todavía no se logra desarrollar la independencia cognoscitiva y el desarrollo del pensamiento reflexivo de los estudiantes en la solución de dichos problemas, se evidencia que existe cierta proyección en las clases para potenciar la relación ciencia profesión, mediante el trabajo científico y las prácticas profesionales, con la inclusión de tareas investigativas que contribuyen al desarrollo del pensamiento reflexivo. Sin embargo no prevalece la creatividad e interés por el descubrimiento, de habilidades para analizar e interpretar problemas, de la comunicación de métodos y la justificación de procesos.

En general, los resultados de la prueba de conocimiento (Recuadro 2) y la prueba de Rigidez del Pensamiento de Davydov (Tabla 1), aplicadas a 12 estudiantes de primer año de la carrera de Ingeniería Hidráulica del curso 2018-2019, de la Universidad de Ciego de Ávila Máximo Gómez Báez; la encuesta que se realizó a 9 profesores de la disciplina Matemática para las carreras de ingeniería de la Universidad de Ciego de Ávila Máximo Gómez Báez (Recuadro 3) y el análisis de 8 controles a clase de Matemática que se realizaron en el departamento de Matemática de la Universidad de Ciego de Ávila Máximo Gómez Báez en el primer semestre del curso 2018-2019, evidencian insuficiencias en la aplicación de procedimientos didácticos para la formación del pensamiento reflexivo desde el proceso de enseñanza aprendizaje de la Matemática en las carreras 
de Ingeniería, la utilización de métodos en las clases que desarrollen el pensamiento reflexivo, la vinculación de los contenidos de las disciplinas con la solución de problemas, lo que dificulta mostrar apropiaciones significativas que incidan en los procesos lógicos reflexivos.

Fundamentos teóricos del Modelo de la dinámica de formación del pensamiento reflexivo En la modelación de la dinámica de la formación del pensamiento reflexivo en el proceso de enseñanza aprendizaje de la Matemática en las carreras de Ingeniería en la Universidad de Ciego de Ávila Máximo Gómez Báez, se asume en el orden epistemológico y metodológico la concepción científica holístico configuracional de Fuentes (2009), que a través de su método holístico dialéctico permite el establecimiento de los eslabones esenciales del proceso modelado, partiendo del reconocimiento de sus configuraciones y las relaciones que se establecen entre estas, que conducen a la transformación del estudiante en su contexto formativo. De esta perspectiva, los eslabones se identifican con niveles de síntesis sucesivas, que expresan los estadios o momentos por el que transita la dinámica del proceso de enseñanza aprendizaje de la Matemática en las carreras de Ingeniería y que al relacionarse e interactuar dialécticamente con otras configuraciones de la misma naturaleza, se integran, formando un todo, que va adquiriendo niveles cualitativamente superiores de organización y efectividad en todo el proceso.

Se establecen los eslabones por los que transita el proceso modelado teniendo los referentes revelados con anterioridad, esencialmente la teoría de Duval (1993), sobre los niveles por los cuales transita el pensamiento reflexivo; las consideraciones de Fuentes (2009), sobre la sistematización formativa, como la vía epistemológica y metodológica para la formación de la capacidad transformadora del sujeto; así como los aportes de Ausubel (1968), en lo relativo a la construcción de los nuevos conocimientos, teniendo en cuenta los previamente adquiridos, entendidos en esta investigación como condición necesaria en el proceso de apropiación de contenidos matemáticos. Fundamentales se consideran además, los presupuestos epistemológicos de la lógica dialéctica planteada por: Lenin (1964), Rodríguez (1983) y Davydov (1986), se constituyen en premisas para fundamentar la necesidad de transitar del pensamiento teórico al pensamiento reflexivo en la formación del pensamiento reflexivo. Estas aportaciones posibilitaron estructurar la lógica de la dinámica de formación del pensamiento reflexivo en el proceso de enseñanza aprendizaje de la Matemática en las carreras de Ingeniería, desde relaciones dialécticas que se establecen entre procesos que se desarrollan en la interactividad heurística interpretativa para la sistematización del contenido matemático y la apropiación de procedimientos lógicos reflexivos.

\section{Modelo de la dinámica de formación del pensamiento reflexivo en el proceso de enseñanza aprendizaje de la Matemática}

Para la construcción del modelo se asume la concepción científica holístico-configuracional de Fuentes (2009), utilizando el método holístico-dialéctico, que permite establecer los eslabones esenciales del proceso modelado, primeramente con un reconocimiento de sus configuraciones, así como las relaciones que se establecen entre estas, lo cual implica transformaciones en el estudiante desde su contexto formativo. Se tiene en cuenta que la lógica de la dinámica de formación del pensamiento reflexivo en el proceso de enseñanza aprendizaje de la Matemática en las carreras de Ingeniería ha transitado desde relaciones dialécticas que se establecen entre procesos que se desarrollan en la interactividad heurística interpretativa para la sistematización del contenido matemático y la apropiación de procedimientos lógicos reflexivos.

Se establecen eslabones que expresan los estadios o momentos por los que transita el proceso modelado, que no se suceden de forma lineal, sino que se interrelacionan en las diferentes formas de enseñanza de esta ciencia para lograr, desde la consecutividad lógica del proceso de enseñanza aprendizaje de la Matemática, la formación del pensamiento reflexivo. Se definen tres eslabones (Figura 1): Establecimiento de las condiciones previas para la formación del pensamiento reflexivo; 
Orientación lógica formal en la formación del pensamiento reflexivo; Aplicación del pensamiento reflexivo en la solución de problemas matemáticos ingenieriles.

Figura 1. Eslabones del Modelo.

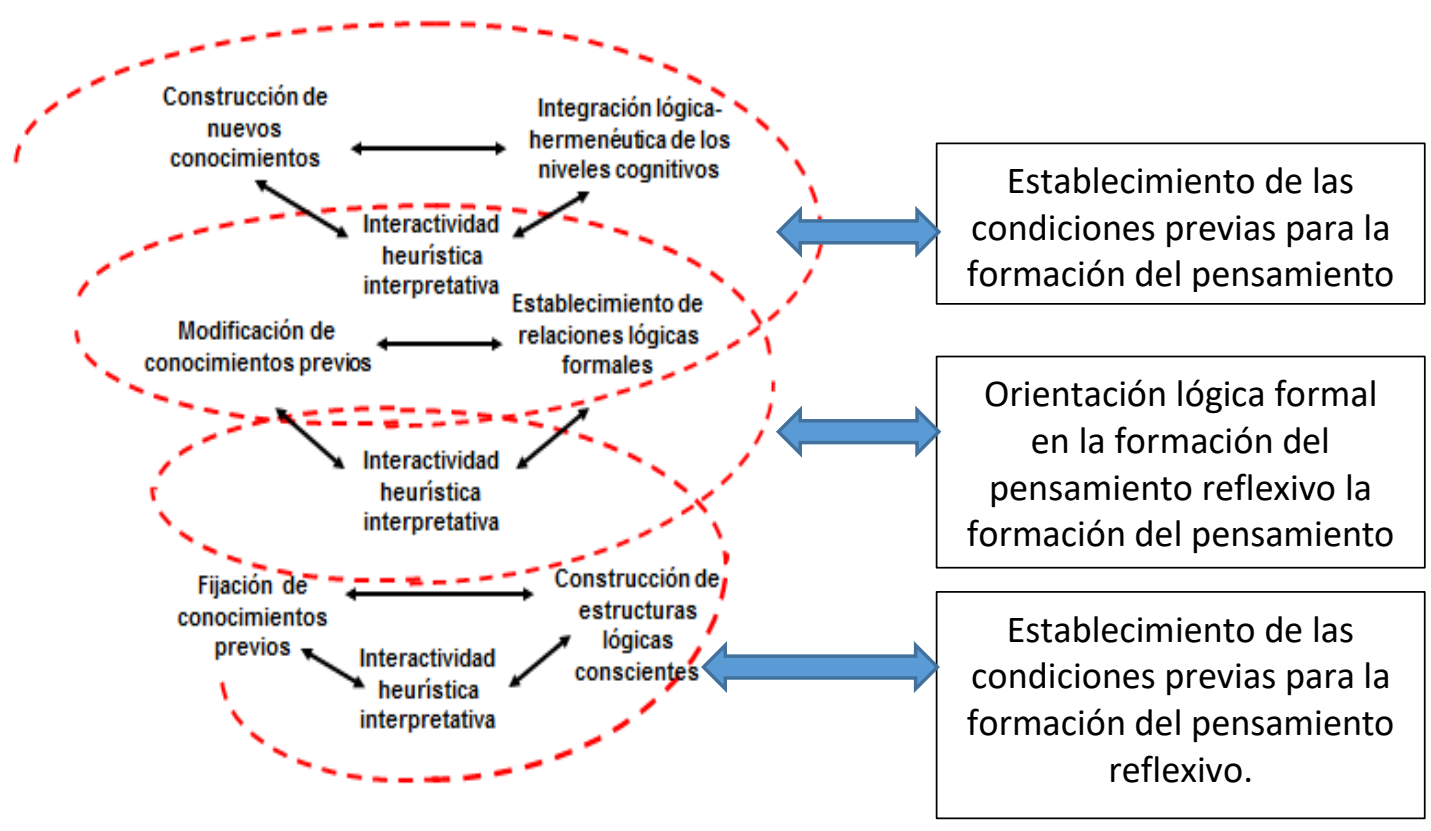

Fuente: Elaboración propia

En la modelación se aportan relaciones dialécticas que fundamentan la necesidad de preparación de las estructuras cognitivas para la apropiación del nuevo contenido, su reconstrucción progresiva y establecimiento, para la solución de problemas matemáticos ingenieriles con un razonamiento lógico, lo que posibilita transitar de forma gradual por diferentes niveles de sistematización de contenidos y de apropiación de procedimientos lógicos reflexivos. Los eslabones expuestos emergen como resultado de la relación dialéctica que se establece entre las configuraciones del proceso modelado. Se diferencian entre sí por las características del quehacer de los sujetos copartícipes en la dinámica de formación del pensamiento reflexivo en el proceso de enseñanza aprendizaje de la Matemática en las carreras de Ingeniería.

1. Establecimiento de las condiciones previas para la formación del pensamiento reflexivo En este eslabón, por una parte surge un cambio de nivel de abstracción, donde se fijan los conocimientos relevantes que ya existen en las estructuras cognitivas, y por la otra se determinan acciones de forma consciente y reflexiva, que se complementan en la interactividad heurística interpretativa, que tiene su expresión en el diálogo que se produce entre todos los actores del proceso de enseñanza aprendizaje de la Matemática, para la búsqueda de un nuevo conocimiento o la solución de problemas matemáticos preparatorios, desde la determinación de contenidos, condiciones y exigencias, en una dialéctica entre la abstracción y la generalización, como procedimientos lógicos asociados a la interpretación.

2. Orientación lógica formal en la formación del pensamiento reflexivo

En este eslabón se modifican los conocimientos previos, se crea una nueva estructura cognitiva en los estudiantes que les permite la solución de problemas matemáticos integradores, donde se combinan los conocimientos preestablecidos con los nuevos, proporcionando la formación del pensamiento reflexivo y con ellos el establecimiento de relaciones lógicas formales desde la interactividad heurística interpretativa que se produce en el proceso de enseñanza aprendizaje de la Matemática. Se fijan los nuevos conocimientos desde una conexión preestablecida con los 
conocimientos relevantes que ya existen en las estructuras cognitivas, en la interactividad heurística interpretativa, mediante la imaginación, percepción de la realidad, el conocimiento implicado; así como las emociones y formas de actuación, lo que permite que el estudiante pueda realizar el análisis del contexto del problema que se presenta, identificar los conocimientos requeridos para su solución y establecer interdependencia entre estos, para desde su aplicación encontrar la solución.

3. Aplicación del pensamiento reflexivo en la solución de problemas matemáticos ingenieriles Este eslabón constituye en sí mismo la interactividad progresiva de acciones para obtener el resultado deseado se produce la construcción de nuevos conocimientos como nueva configuración de la dinámica de formación del pensamiento reflexivo en el proceso de enseñanza aprendizaje de la Matemática en las carreras de Ingeniería, que expresa un elevado nivel de profundidad en la función reguladora de la metacognición, su naturaleza y características para lograr seleccionar una solución adecuada a los problemas matemáticos ingenieriles, lo que conlleva a la consolidación de los niveles superiores de la reflexión, mediante una reorganización progresiva de los procesos cognitivos resultantes de la maduración biológica y la experiencia adquirida por el estudiante.

Teniendo en cuenta el análisis realizado de cómo transcurre la dinámica de formación del pensamiento reflexivo en el proceso de enseñanza aprendizaje de la Matemática en las carreras de Ingeniería en la Universidad de Ciego de Ávila Máximo Gómez Báez y las cualidades esenciales reveladas, se definen tres relaciones fundamentales, que se ponen de manifiesto en este proceso formativo (Figura 2): Relación de preparación de las estructuras cognitivas para la apropiación del nuevo contenido; Relación de reconstrucción progresiva de las estructuras cognitivas mediante la integración de conocimientos anteriores y posteriores; Relación de establecimiento de las estructuras cognitivas para la solución de problemas matemáticos ingenieriles con un pensamiento reflexivo.

Figura 2. Relaciones fundamentales en el Modelo

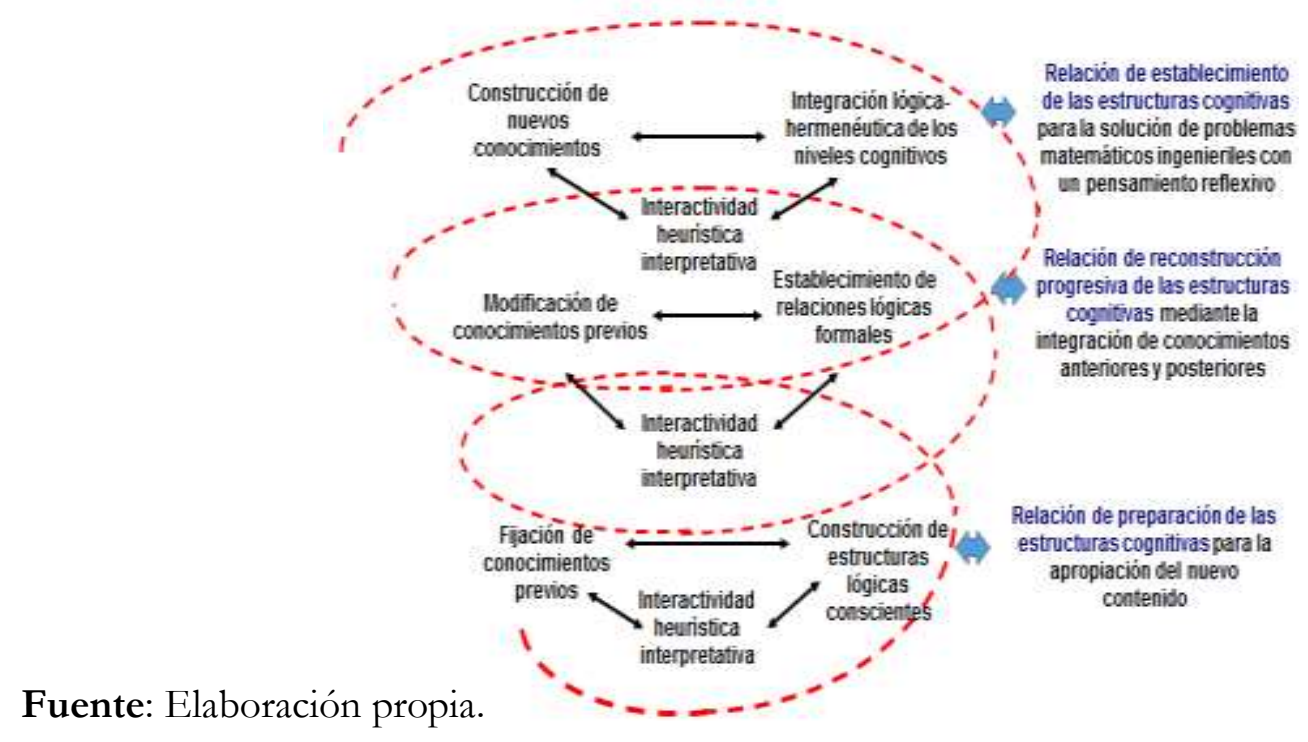

La regularidad fundamental del modelo se concreta el carácter de la interactividad heurística interpretativa que se desarrolla en la dinámica de formación del pensamiento reflexivo en el proceso de enseñanza aprendizaje de la Matemática en las carreras de Ingeniería, desde la lógica integradora que se produce entre la sistematización de contenidos y la apropiación de procedimientos lógicos reflexivos desde los niveles por los que transita el proceso formativo modelado. 
Sistema de procedimientos didácticos para la formación del pensamiento reflexivo desde el proceso de enseñanza aprendizaje de la Matemática en las carreras de ingeniería.

Para la construcción del Sistema de procedimientos didáctico se tiene en cuenta la lógica de la dinámica de formación del pensamiento reflexivo en el proceso de enseñanza aprendizaje de la Matemática en las carreras de Ingeniería, desde relaciones dialécticas que se establecen entre procesos que se desarrollan en la interactividad heurística, hacia la sistematización del contenido matemático y la apropiación de procedimientos lógicos reflexivos. Los procedimientos, que posee el sistema, no se consideran etapas preestablecidas, sino niveles de construcción dinámica que contemplan la posibilidad de adaptación y enriquecimiento en la praxis pedagógica, de manera que su ejecución no sigue un orden lineal, se suceden en el proceso de enseñanza aprendizaje de la Matemática para las carreras de Ingeniería en sus diferentes formas y tipologías de enseñanza. Estos procedimientos se definen teniendo en cuenta los factores contextuales que condicionan el desarrollo del proceso, y aquellas cualidades que explican y singularizan una lógica en el movimiento del objeto. Para construir el Sistema de procedimientos didácticos para el proceso de enseñanza aprendizaje de la Matemática en las carreras de Ingeniería se delimitan aspectos ilustrados en la Figura 3.

Figura 3. Sistema de procedimientos didácticos para la formación del pensamiento reflexivo desde el proceso de enseñanza aprendizaje de la Matemática en carreras de Ingeniería de la Universidad de Ciego de Ávila Máximo Gómez Báez

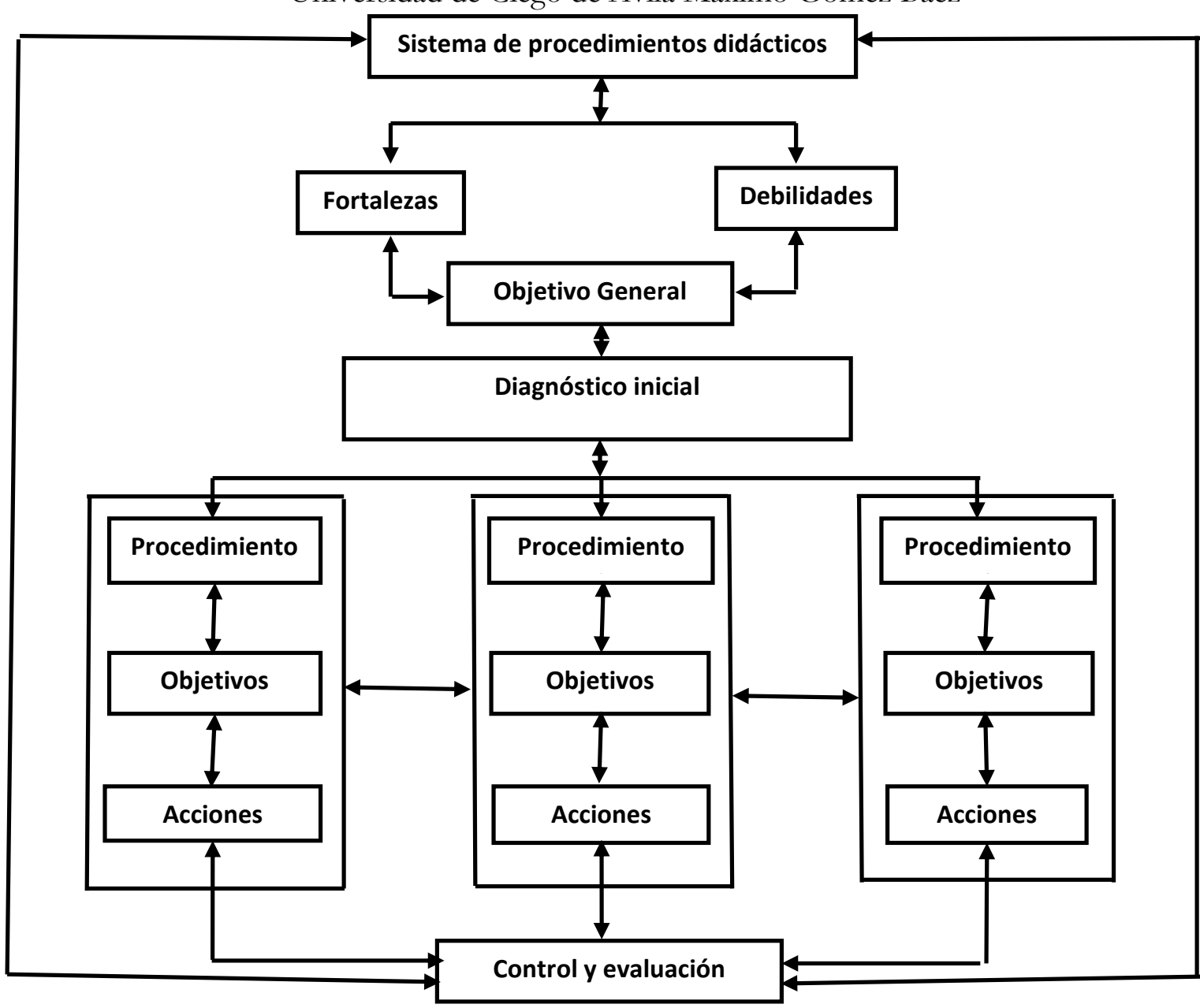

Fuente: Elaboración propia 
En este sentido, deberán precisarse las siguientes fortaleza: el plan de estudio de las carreras de Ingeniería está estructurado y actualizado por la Comisión Nacional de carreras; en el programa de Matemática para las carreras de Ingeniería se constata como exigencia la formación del pensamiento reflexivo en los estudiantes; se cuenta con un colectivo de departamento con experiencia en la docencia de la disciplina de Matemática; en la Universidad de Ciego de Ávila "Máximo Gómez Báez", las aulas y laboratorios de computación están preparadas para el pertinente desarrollo del proceso de enseñanza aprendizaje de los estudiantes; se dispone de una plataforma virtual para la interacción con los estudiantes y la orientación de la auto preparación. Se precisan las siguientes debilidades: no todos los profesores del colectivo de la disciplina Matemática para las carreras de Ingeniería están preparados para formar al estudiante con un pensamiento reflexivo; se desarrollan limitadas actividades metodológicas dirigidas a la preparación profesoral con vista a la formación del pensamiento reflexivo en los estudiantes; los problemas de los libros de texto comúnmente no se corresponden con el perfil ingenieril.

El objetivo general que se pretende desde las acciones implementadas en cada procedimiento es el consejo instructivo de forma preconcebida del proceso de formación del pensamiento reflexivo desde el proceso de enseñanza aprendizaje de la Matemática en las carreras de Ingeniería.

Diagnóstico inicial

La determinación de la problemática existente y la detección de la situación real que se presenta en los estudiantes que cursan las carreras de Ingeniería, se consideran esenciales para precisar el estado en que se encuentra la formación del pensamiento reflexivo en el proceso de enseñanza aprendizaje de la Matemática. En este momento es fundamental identificar la disponibilidad de conocimientos y habilidades en las estructuras cognitivas de cada estudiante, así como el grado de desarrollo del pensamiento, para ello se realiza la caracterización del estado actual de la formación del pensamiento reflexivo desde el proceso de enseñanza aprendizaje de la Matemática en las carreras de Ingeniería, detallado con anterioridad. Los indicadores evaluativos para la realización del diagnóstico de la situación que presentan los estudiantes son: comprensión de los problemas propuestos; selección de las alternativas de solución de los problemas propuestos; aplicación de los métodos seleccionados en el proceso de solución de los problemas propuestos; argumentación y valoración de los resultados en la solución de los problemas propuestos.

Estos indicadores son de suma importancia a la hora de implementar las acciones propuestas en el Sistema de procedimientos didácticos ya que posibilitan a las personas incluidas en este proceso, el reconocimiento de elementos fundamentales que están siendo endebles en la formación del pensamiento reflexivo en la disciplina Matemática en las carreras de Ingeniería, así como entender las dificultades que tienen los estudiantes respecto a la indagación de nuevos métodos como alternativas para darle solución a problemas matemáticos profesionales.

Para la realización de este diagnóstico, se implementa la caracterización del estado actual de la formación del pensamiento reflexivo desde el proceso de enseñanza aprendizaje de la Matemática en las carreras de Ingeniería, explicada con anterioridad.

\section{Determinación de los procedimientos}

\section{Procedimiento 1. Establecimiento de condiciones previas para la formación del pensamiento reflexivo}

Objetivo específico: Establecer las condiciones para la formación del pensamiento reflexivo desde la fijación de conocimientos previos y construcción de estructuras lógicas conscientes en la interactividad heurística interpretativa que se desarrolla en el proceso de enseñanza aprendizaje de la Matemática.

Acciones para el profesor: determinar los conocimientos mínimos exigibles para la apropiación de los nuevos contenidos, mediante el estudio del programa de la disciplina Matemática para las carreras de Ingeniería; diagnosticar, antes de impartir cada contenido nuevo, el nivel de conocimiento de los estudiantes, como premisa para la apropiación de los nuevos contenidos; 
formular los objetivos de las clases, donde se tenga en cuenta qué parte del contenido precedente es preciso dominar por parte del estudiante; estructurar los contenidos, en las clases, partiendo de los conocimientos mínimos exigibles que ya existen en las estructuras cognitivas de los estudiantes; propiciar en las clases una conversación heurística que lleve a los estudiantes a reflexionar acerca de los conocimientos previos pertinentes para la comprensión de los nuevos contenidos; exigir durante las clases la identificación de los conocimientos previos requeridos en la solución de problemas matemáticos; orientar la solución de problemas matemáticos preparatorios, donde se apliquen los conocimientos preestablecidos en correspondencia con los ritmos de aprendizaje de los estudiantes; crear equipos de trabajo para la cooperación en la fijación de conocimientos previos requeridos para la comprensión de los nuevos contenidos.

Acciones para el estudiante: autoevaluar el dominio de los conocimientos previos requeridos para la comprensión de los nuevos contenidos; resolver problemas matemáticos preparatorios de forma individual y en equipos para la fijación de los conocimientos previos requeridos en la comprensión de nuevos contenidos; solicitar al profesor o a sus compañeros explicación sobre posibles dudas sobre conocimientos mínimos exigibles; debatir en equipos de trabajo la solución de problemas matemáticos preparatorios.

\section{Procedimiento 2. Orientación lógica formal en la formación del pensamiento reflexivo}

Objetivo específico: Desarrollar una orientación lógica formal en la formación del pensamiento reflexivo de los estudiantes, desde la transformación de conocimientos previos y establecimiento de relaciones lógicas formales en la interactividad heurística interpretativa que se extiende en el proceso de enseñanza aprendizaje de la Matemática.

Acciones específicas: Planificar las clases, estableciendo relaciones entre los conocimientos mínimos exigibles y los nuevos conocimientos para facilitar la comprensión, propiciar en las clases una conversación heurística que lleve a los estudiantes a la reflexión sobre la relación existente entre conocimientos previos y nuevos contenidos, destacando el valor de los primeros para la comprensión; orientar la solución de problemas matemáticos integradores, diferenciados en correspondencia con los niveles de aprendizaje de los estudiantes; discutir en las actividades la solución de los problemas planteados, donde los estudiantes identifiquen los conocimiento previos y los conocimientos nuevos requeridos.

Acciones para el estudiante: resolver problemas matemáticos integradores de forma individual y en equipos para la fijación de los conocimientos previos pertinentes en la aprehensión de nuevos contenidos; solicitar al profesor o a sus compañeros explicación sobre posibles dudas sobre conocimientos la solución de problemas integradores; debatir en equipos de trabajo la solución de problemas matemáticos integradores.

\section{Procedimiento 3. Aplicación del pensamiento reflexivo en la solución de problemas contextualizados}

Objetivo específico: Aplicar el pensamiento reflexivo en la solución de problemas matemáticos contextualizados sobre la base de construcción de nuevos conocimientos e integración lógica hermenéutica de niveles cognitivos en la interactividad heurística interpretativa que se extiende en el proceso de enseñanza aprendizaje de la Matemática.

Acciones para el profesor: proponer en las clases prácticas la solución de problemas matemáticos ingenieriles, que requieran de la implementación de los conocimientos matemáticos adquiridos; exigir en las clases prácticas la argumentación del proceso de solución de los problemas matemáticos ingenieriles desde la integración de los niveles cognitivos; orientar tareas individuales y en equipo, sobre la solución de problemas matemáticos ingenieriles; incluir progresivamente la aplicación de asistentes matemáticos para la solución de problemas y como vía de verificación de los resultados; realizar seminarios donde los estudiantes presenten tareas de investigación sobre determinados temas y pongan de manifiesto el ordenamiento lógico de los contenidos; realizar 
talleres donde los estudiantes expongan los resultados de la solución de problemas matemáticos ingenieriles identificados en sus esferas de actuación, a partir del vínculo entre los componentes académico, investigativo y laboral.

Acciones para el estudiante: resolver problemas matemáticos ingenieriles, que requieran de la aplicación de los conocimientos adquiridos en las diferentes tipologías de clase y en tareas extra clases; realizar tareas investigativas orientadas por el profesor, individuales y por equipos, donde pongan de manifiesto el ordenamiento lógico de los contenidos en sus estructuras cognitivas; presenta el resultado de las tareas en seminarios; visitar empresas vinculadas a sus esferas de actuación para identificar problemas matemáticos ingenieriles; exponer en talleres la solución de problemas matemáticos ingenieriles.

Orientaciones metodológicas: se realizan reuniones del colectivo de disciplina para revisar el programa de la disciplina e identificar el sistema de conocimientos mínimos exigibles por asignaturas y temas, así como para identificar las dificultades que van a presentar los estudiantes; se diseñan en el colectivo de disciplina problemas matemáticos preparatorios, integradores e ingenieriles para el trabajo con los estudiantes en correspondencia con las necesidades individuales; se utilizan tipologías de clases como conferencias, clases prácticas, seminarios, talleres; en las conferencias se utiliza el método expositivo problémico y la conversación heurística para que el estudiante siempre se encuentre en una posición activa de reflexión constante en la construcción del nuevo conocimiento sobre la base de lo conocido; se estructuran las actividades de clases prácticas, seminarios y talleres de manera que se propicie el tránsito de los estudiantes por los niveles de asimilación; en las clases prácticas se utilizan métodos de elaboración conjunta y se transita paulatinamente al trabajo independiente; en las clases se organizan actividades por equipo y de forma individual; se utilizan materiales elaborados por el profesor para proponer a los estudiantes problemas matemáticos preparatorios y libros de texto de la enseñanza media superior; se utilizan materiales elaborados por el profesor para proponer a los estudiantes problemas matemáticos integradores e ingenieriles, así como los textos de la asignatura.

\section{Evaluación de los resultados}

Se realiza un control sistemático del cumplimiento de las acciones del Sistema de procedimientos didácticos durante todo el proceso de enseñanza aprendizaje de la Matemática y al concluir cada semestre. Se evalúa el cumplimiento de las metas para cada estadio, lo que posibilita constatar el grado de cumplimiento del objetivo y de las acciones programadas para cada procedimiento.

Indicadores para evaluar la pertinencia en la aplicación del Sistema de procedimientos didácticos: grado de cumplimiento de los objetivos en cada uno de los procedimientos y en general; grado de cumplimiento de las acciones en cada uno de los procedimientos; métodos que prevalecen en el proceso de enseñanza aprendizaje de la Matemática; vías utilizadas para el desarrollo del pensamiento reflexivo en la enseñanza aprendizaje de la Matemática; relación ciencia profesión en el proceso enseñanza aprendizaje de la Matemática.

La aplicación del Sistema de procedimientos didácticos conlleva a potenciar la formación del pensamiento reflexivo. Para medir las transformaciones logradas, se tienen en cuenta los siguientes indicadores: selección de las alternativas de solución de los problemas propuestos; aplicación de los métodos seleccionados en el proceso de solución de los problemas propuestos; argumentación y valoración de los resultados en la solución de los problemas propuestos.

Para comprobar las transformaciones producidas en la formación del pensamiento reflexivo, se seleccionaron una muestra intencional 12 estudiantes, que cursaron primer año de la carrera de Ingeniería Hidráulica de la Universidad de Ciego de Ávila Máximo Gómez Báez, en los cursos 2018-2019, 2019-2020, que transita desde el primer año de la carrera hasta el segundo año, al inicio (como se mostró anteriormente en la caracterización del estado actual de la formación del pensamiento reflexivo desde el proceso de enseñanza aprendizaje de la Matemática en carreras de Ingeniería), se le aplicó a los estudiantes tomados como muestra la prueba de conocimiento 
(Recuadro 2), los resultados del análisis estadístico descriptivo se encuentran en la Tabla 2, posteriormente se revisaron las pruebas finales de Matemática aplicadas a los estudiantes tomados como muestra en los años 2019 y 2020 (Recuadro 5) y se realizó un análisis estadístico descriptivo de estas pruebas (Tablas 4 y 5), con el objetivo de evaluar en los estudiantes la formación del pensamiento reflexivo.

Tabla 4. Resultado de la Prueba Final de Matemática 2019 que se aplicó a los estudiantes de Ingeniería Hidráulica

\begin{tabular}{|ll|l|l|l|l|}
\hline & Frecuencia & Porcentaje & $\begin{array}{l}\text { Porcentaje } \\
\text { válido }\end{array}$ & $\begin{array}{l}\text { Porcentaje } \\
\text { acumulado }\end{array}$ \\
\hline \multirow{4}{*}{ Válidos } & Bajo & 3 & 25,0 & 25,0 & 25,0 \\
& Medio & 5 & 41,7 & 41,7 & 66,7 \\
& Alto & 4 & 33,3 & 33,3 & 100,0 \\
& Total & 12 & 100,0 & 100,0 & \\
\hline
\end{tabular}

Fuente: Elaboración propia

Tabla 5. Resultado de la Prueba Final de Matemática 2020 que se aplicó a los 12 estudiantes de la carrera de Ingeniería Hidráulica de la Universidad de Ciego de Ávila

\begin{tabular}{|c|c|c|c|c|c|}
\hline & & Frecuencia & Porcentaje & $\begin{array}{l}\text { Porcentaje } \\
\text { válido }\end{array}$ & $\begin{array}{l}\text { Porcentaje } \\
\text { acumulado }\end{array}$ \\
\hline \multirow{5}{*}{ Válidos } & Medio & 3 & 25,0 & 25,0 & 25,0 \\
\hline & Alto & 8 & 66,7 & 66,7 & 91,7 \\
\hline & Muy & 1 & 83 & 83 & 1000 \\
\hline & Alto & & & & \\
\hline & Total & 12 & 100,0 & 100,0 & \\
\hline
\end{tabular}

Fuente: Elaboración propia

Recuadro 5. Guía de análisis documental de las pruebas finales de Matemática 2019 y 2020 aplicadas a los 12 estudiantes de la carrera de Ingeniería Hidráulica de la Universidad de Ciego de Ávila

Objetivo: Evaluar el dominio de los contenidos estudiados de Matemática en los estudiantes de la Carrera Ingeniería Hidráulica.

Universo: Temarios de pruebas finales de Matemática del 2019 y 2020 aplicadas en la carrera de Ingeniería Hidráulica.

Tipo de estudio documental: Análisis de contenido.

Tipo de documentos: Escritos.

Categorías de análisis:

- Grado de precisión en la selección de las alternativas de solución del problema propuesto.

- Precisión en la aplicación de los métodos para la solución del problema.

- Niveles de argumentación.

Fuente: Elaboración propia

Como se mostró anteriormente en la caracterización del estado actual de la formación del pensamiento reflexivo desde el proceso de enseñanza aprendizaje de la Matemática en carreras de Ingeniería, se le aplicó a los estudiantes tomados como muestra la prueba de conocimiento (Recuadro 2), los resultados del análisis estadístico descriptivo se encuentran en la Tabla 2, posteriormente se revisaron las pruebas finales de Matemática aplicadas a los estudiantes tomados 
como muestra en los años 2019 y 2020 (Recuadro 5) y se realizó un análisis estadístico descriptivo de estas pruebas (Tablas 4 y 5), con el objetivo de evaluar en los estudiantes la formación del pensamiento reflexivo.

Para corroborar las transformaciones de los 12 estudiantes que se seleccionaron como muestra, se realizó la prueba no paramétrica de W de Kendall (Tabla 6), para muestras relacionadas, en la que se comparó el comportamiento de las pruebas: Prueba de conocimiento, analizada anteriormente en la caracterización del estado actual de la formación del pensamiento reflexivo desde el proceso de enseñanza aprendizaje de la Matemática en carreras de Ingeniería (Recuadro 2), Prueba final de Matemática 2019 (Recuadro 5) y Prueba final de Matemática 2020 (Recuadro 5), aplicadas a los 12 estudiantes tomados como muestra, las Hipótesis: nula $\left(\mathrm{H}_{\mathrm{o}}\right)$ y alternativa $\left(\mathrm{H}_{1}\right)$ se declararon de la forma siguiente:

$\mathrm{H}_{0}$ : No existen diferencias significativas entre los resultados en la prueba de conocimiento, la prueba final de Matemática 2019 y la prueba final de Matemática 2020.

$\mathrm{H}_{1}$ : Existen diferencias significativas entre los resultados en la prueba de conocimiento, la prueba final de Matemática 2019 y la prueba final de Matemática 2020.

$\mathrm{Al}$ procesar la información se toma $\alpha=0.05$ y se obtiene $\mathrm{p}=0.000$, por tanto la hipótesis nula se rechaza ( $p$ es menos que 0,05 ), se puede concluir que hay diferencias significativas en los resultados de las tres pruebas aplicadas a los estudiantes seleccionados como muestra.

Tabla 6. Resultados de la prueba no paramétrica de W de Kendall

Fuente: Elaboración propia

Prueba W de Kendall

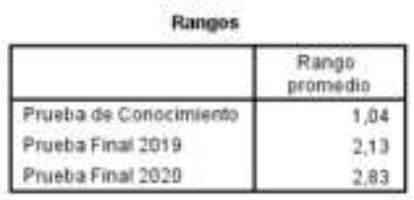

Estadisticos de contraste

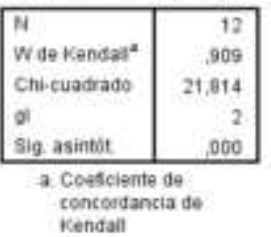

Kendail

Como valoración cualitativa del proceso llevado a cabo, basado en la experiencia y en la observación de los hechos, en el progresar de la aplicación del Sistema de procedimientos didácticos, los estudiantes fueron capaces de: analizar las diferentes alternativas de solución y seleccionar la más acertada; hacer énfasis en encontrar la alternativa de solución óptima; aminorar la propensión en los estudiantes predominada por esquemas reproductivos, así como argüir la evaluación de los resultados de la solución de los problemas matemáticos.

Como evaluación cualitativa del proceso implementado, se pudo confirmar empíricamente, que desde el momento que se ejecutó el Sistema de procedimientos didácticos, los estudiantes, tienen tendencia a:

- Analizar las diferentes alternativas de solución y escoger la más acertada.

- Hacer énfasis en encontrar la alternativa de solución óptima.

- Disminuir la tendencia primada por esquemas reproductivos.

- Discutir la valoración de los resultados en la solución de los problemas. 


\section{Valoración de la pertinencia del Sistema de procedimientos didácticos mediante Criterio de Expertos}

Para realizar la valoración cualitativa de los resultados del Modelo y del Sistema de procedimientos didácticos se utilizó el Criterio de Expertos, seleccionando 16 posibles expertos, todos Doctores en Ciencias de la Educación Superior y de profesores titulares con más de 20 años de experiencia docente en las carreras de Ingeniería, donde 5 son Ingenieros y 11 son Matemáticos. A los 16 posibles expertos se les aplicó la encuesta que aparece en el Tabla 7. Los resultados obtenidos posibilitaron revelar el grado de conocimiento de cada posible experto sobre la temática investigada y las fuentes de argumentación de estos conocimientos, determinando así la competencia de cada posible Experto (Tabla 8).

Tabla 7. Consulta a los 16 posibles expertos.

Datos de los posibles Expertos:

Formación profesional:

Ocupación actual:

Experiencia en la Educación Superior:

Marque con una cruz las fuentes que usted considera que han influido en su conocimiento sobre el tema, en un grado alto, medio o bajo.

\begin{tabular}{|l|l|l|l|}
\hline \multirow{2}{*}{ Fuentes de argumentación } & \multicolumn{3}{|l|}{$\begin{array}{l}\text { Grado de influencia de } \\
\text { cada una de las fuentes } \\
\text { en sus criterios }\end{array}$} \\
\cline { 2 - 4 } & $\begin{array}{l}\text { A } \\
\text { (Alto }\end{array}$ & $\begin{array}{l}\text { M } \\
\text { (Medio }\end{array}$ & $\begin{array}{l}\text { B } \\
\text { (Bajo) }\end{array}$ \\
\hline Análisis teóricos realizados (A.T.) & & & \\
\hline Experiencia como profesional (E. O.) & & & \\
\hline Trabajos de autores nacionales (A. N.) & & & \\
\hline Trabajos de autores extranjeros (A. E.) & & & \\
\hline $\begin{array}{l}\text { Sus propios conocimientos sobre el } \\
\text { estado del problema de investigación } \\
\text { (P. C.) }\end{array}$ & & & \\
\hline Su intuición (I.) & & & \\
\hline
\end{tabular}

Fuente: Elaboración propia

Tabla 8. Competencia de los 16 posibles expertos

\begin{tabular}{|l|l|l|l|l|l|}
\hline $\begin{array}{l}\text { Especialista } \\
\text { s }\end{array}$ & $\mathrm{Kc}$ & $\mathrm{Ka}$ & $\mathrm{K}=\frac{\mathrm{Kc}+\mathrm{Ka}}{2}$ & Clasificación & $\begin{array}{l}\text { Experto } \\
\mathrm{s}\end{array}$ \\
\hline 1. & 0,8 & 0,9 & 0,85 & alto & $\mathrm{X}$ \\
\hline 2. & 0,8 & 0,9 & 0,85 & alto & $\mathrm{X}$ \\
\hline 3. & 0,9 & 0,9 & 0,9 & alto & $\mathrm{X}$ \\
\hline 4. & 1 & 0,9 & 0,95 & alto & $\mathrm{X}$ \\
\hline 5. & 0,7 & 0,8 & 0,75 & medio & $\mathrm{X}$ \\
\hline 6. & 0,7 & 0,8 & 0,75 & medio & $\mathrm{X}$ \\
\hline 7. & 0,8 & 0,9 & 0,85 & alto & $\mathrm{X}$ \\
\hline 8. & 0,6 & 0,6 & 0,6 & medio & $\mathrm{X}$ \\
\hline 9. & 0,6 & 0,8 & 0,7 & medio & $\mathrm{X}$ \\
\hline 10. & 0,7 & 0,8 & 0,75 & medio & $\mathrm{X}$ \\
\hline 11. & 0,9 & 0,6 & 0,75 & medio & $\mathrm{X}$ \\
\hline 12. & 0,8 & 0,9 & 0,85 & alto & $\mathrm{X}$ \\
\hline
\end{tabular}




\begin{tabular}{|l|l|l|l|l|l|}
\hline 13. & 0,6 & 0,5 & 0,55 & medio & $\mathrm{X}$ \\
\hline 14. & 0,7 & 0,8 & 0,75 & medio & $\mathrm{X}$ \\
\hline 15. & 0,7 & 0,8 & 0,75 & medio & $\mathrm{X}$ \\
\hline 16. & 0,8 & 0,9 & 0,85 & alto & $\mathrm{X}$ \\
\hline
\end{tabular}

$\mathrm{Ka}$ - Coeficiente de argumentación o fundamentación de sus conocimientos

$\mathrm{Kc}$ - Coeficiente de conocimiento o información del experto

$\mathrm{K}$ - Coeficiente de competencia

Si $0,8<\mathrm{K} \leq 1,0$; entonces el coeficiente de competencia es alto.

Si $0,5<K \leq 0,8$; entonces el coeficiente de competencia es medio.

Si $\mathrm{K} \leq 0,5$; entonces el coeficiente de competencia es bajo.

Fuente: Elaboración propia

Teniendo en cuenta los resultados obtenidos, se determinó escoger los 16 expertos propuestos, los cuales poseen competencia media o alta, como se muestra en la Tabla 8. Para conocer el grado de pertinencia del Modelo de la dinámica de formación del pensamiento reflexivo en el proceso de enseñanza aprendizaje de la Matemática en las carreras de Ingeniería y del Sistema de procedimientos didácticos para el proceso de enseñanza aprendizaje de la Matemática en las carreras de Ingeniería, de la Universidad de Ciego de Ávila Máximo Gómez Báez, se le entrega a los expertos un documento, con la descripción de estos aportes (Modelo y Sistema de Procedimientos Didácticos). Primeramente, se les pide por escrito sus opiniones y sus sugerencias sobre la propuesta, dándole un plazo para que elaboren sus ideas. En la primera vuelta se perfecciona la propuesta utilizando los datos que aportaron los expertos, posteriormente se realiza una nueva consulta mediante una encuesta (Tabla 9). Los resultados de los diferentes aspectos a evaluar aparecen en la Tabla 10 y 11.

Tabla 9. Encuesta a los 16 Expertos seleccionados

Objetivo de la encuesta: Valorar la pertinencia científico-metodológica del Modelo de la dinámica de formación del pensamiento reflexivo en el proceso de enseñanza aprendizaje de la Matemática en las carreras de Ingeniería y del Sistema de procedimientos didácticos para el proceso de enseñanza aprendizaje de la Matemática en las carreras de Ingeniería.

\begin{tabular}{|l|l|l|l|l|l|}
\hline Indicadores & $\begin{array}{l}\text { Muy } \\
\text { Adecuada } \\
(5)\end{array}$ & $\begin{array}{l}\text { Bastante } \\
\text { Adecuada } \\
(4)\end{array}$ & $\begin{array}{l}\text { Adecuada } \\
(3)\end{array}$ & $\begin{array}{l}\text { Poco } \\
\text { Adecuada } \\
(2)\end{array}$ & $\begin{array}{l}\text { No } \\
\text { Adecuad } \\
\text { a (1) }\end{array}$ \\
\hline $\begin{array}{l}\text { El modelo constituye un aporte novedoso, } \\
\text { que contribuye a enriquecer la Didáctica de } \\
\text { la Matemática para carreras de Ingeniería. }\end{array}$ & & & & & \\
\hline $\begin{array}{l}\text { Los fundamentos epistemológicos, que } \\
\text { apoyan la construcción teórica, permiten } \\
\text { revelar aquellas categorías que contribuyen } \\
\text { a comprender y explicar la esencia del } \\
\text { proceso de formación del pensamiento } \\
\text { reflexivo en estudiantes de las carreras de } \\
\text { Ingeniería. }\end{array}$ & & & & & \\
\hline $\begin{array}{l}\text { Las configuraciones del modelo } \\
\text { constituyen procesos que en su } \\
\text { interrelación posibilitan explicar el proceso } \\
\text { de formación del pensamiento reflexivo } \\
\text { desde este proceso formativo con una } \\
\text { orientación hacia la solución de problemas. }\end{array}$ & & & & & \\
\hline $\begin{array}{l}\text { El Sistema de Procedimientos Didácticos } \\
\text { del proceso de enseñanza aprendizaje de la }\end{array}$ & & & & & \\
\hline
\end{tabular}




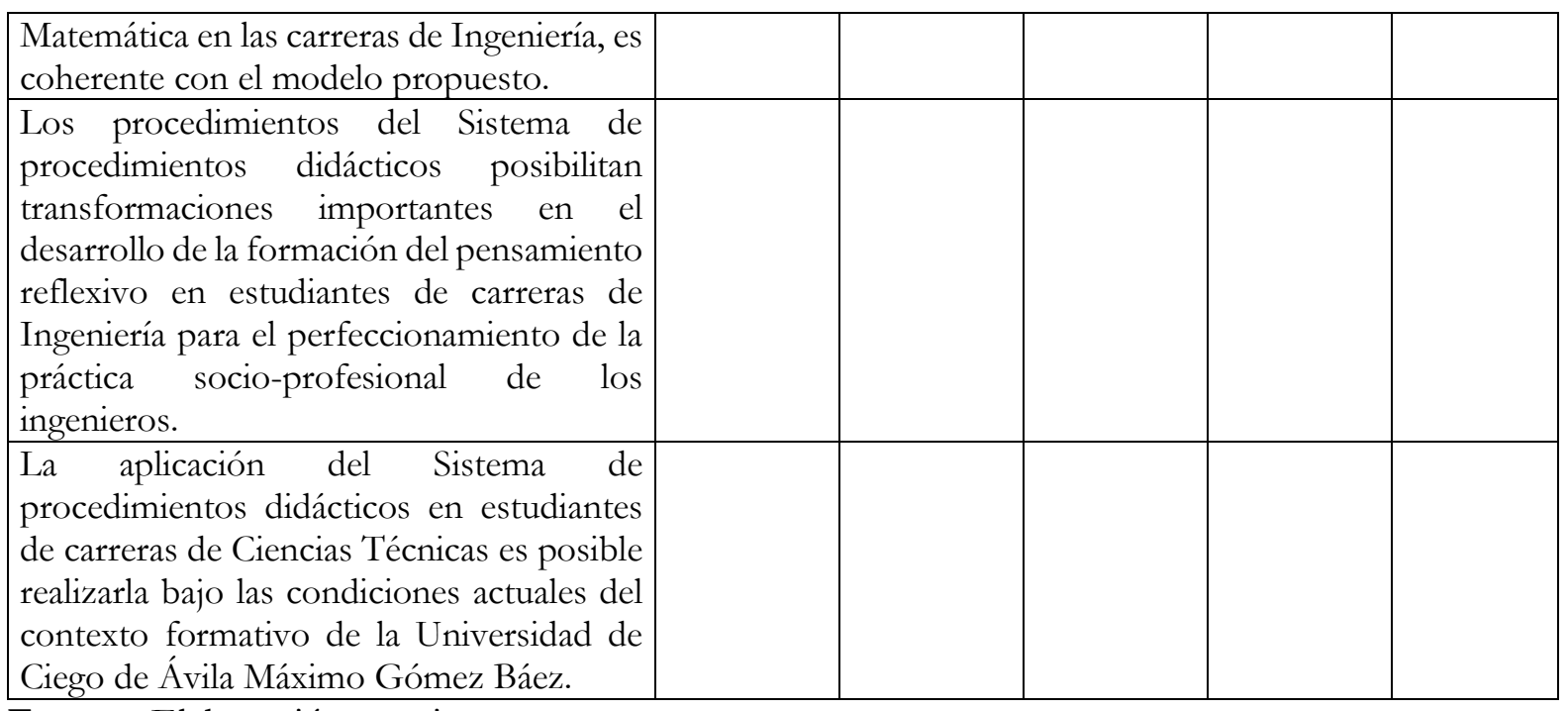

Fuente: Elaboración propia

Tabla 10. Resultado de las encuestas que se aplicaron a los 16 Expertos. La E representa

\begin{tabular}{|l|l|l|l|l|}
\hline Muy & Bastante & & Poco & No \\
Adecuada & Adecuad & Adecuada & Adecuada & Adecuad \\
$(5)$ & a (4) & $(3)$ & $(2)$ & a (1) \\
\hline M.A & B.A & A & P.A & N.A \\
\hline
\end{tabular}

\begin{tabular}{|l|l|l|l|l|l|l|}
\hline \multirow{2}{*}{ Expertos } & \multicolumn{5}{|l|}{ Categorías (Aspectos a evaluar) } \\
\cline { 2 - 7 } & I1 & I2 & I3 & I4 & I5 & I6 \\
\hline E1 & M.A & B.A & B.A & M.A & B.A & A \\
\hline E2 & M.A & B.A & M.A & M.A & M.A & M.A \\
\hline E3 & M.A & M.A & M.A & M.A & M.A & M.A \\
\hline E4 & M.A & B.A & B.A & B.A & B.A & B.A \\
\hline E5 & M.A & M.A & M.A & B.A & M.A & M.A \\
\hline E6 & M.A & M.A & M.A & B.A & M.A & A \\
\hline E7 & B.A & M.A & M.A & M.A & M.A & M.A \\
\hline E8 & M.A & M.A & B.A & M.A & M.A & M.A \\
\hline E1 & M.A & B.A & M.A & M.A & M.A & B.A \\
\hline E10 & M.A & M.A & M.A & M.A & M.A & M.A \\
\hline E11 & B.A & B.A & B.A & B.A & B.A & B.A \\
\hline E12 & B.A & B.A & M.A & B.A & B.A & M.A \\
\hline E13 & M.A & B.A & B.A & A & B.A & M.A \\
\hline E14 & M.A & M.A & M.A & M.A & M.A & M.A \\
\hline E15 & M.A & M.A & M.A & M.A & M.A & M.A \\
\hline E16 & B.A & B.A & B.A & B.A & B.A & B.A \\
\hline
\end{tabular}

Fuente: Elaboración propia 
En esta Tabla 10, se puede observar que existe consenso entre los expertos en cuanto al aporte novedoso que tiene el modelo, donde estos plantean que: en sus fundamentos se revelan categorías que contribuyen a comprender y explicar la esencia del proceso de formación del pensamiento reflexivo en estudiantes de las carreras de Ingeniería; las configuraciones del modelo constituyen procesos que en su interrelación posibilitan explicar este proceso formativo; el Sistema de procedimientos didácticos es coherente con el modelo propuesto; sus acciones posibilitan transformaciones importantes en los estudiantes y son factibles de introducir bajo las condiciones actuales del contexto formativo de la Universidad de Ciego de Ávila Máximo Gómez Báez., al ser evaluados todos los aspectos de muy adecuados o bastante adecuados (Tabla 11). Algunas de las sugerencias planteadas por los expertos fueron: lograr una correspondencia entre el proceso de enseñanza aprendizaje de la Matemática y el proceso de enseñanza aprendizaje de las asignaturas de la especialidad, con vista a aplicar la Matemática al perfil Ingenieril mediante la solución de problemas contextualizados a la realidad ingenieril.

Tabla 11. Resultados, por indicadores de la encuesta que se realizó a los 16 Expertos.

\begin{tabular}{|c|c|c|c|c|c|c|c|c|}
\hline \multicolumn{8}{|c|}{ Cálculo de puntos de corte y escala de los Indicadores } & \multirow[b]{2}{*}{ Resultado } \\
\hline Aspectos & \begin{tabular}{|l|} 
Muy \\
Adecuado
\end{tabular} & \begin{tabular}{|l|} 
Bastante \\
Adecuado \\
\end{tabular} & Adecuado & \begin{tabular}{|l|} 
Poco \\
Adecuado \\
\end{tabular} & Suma & Promedio & N-Prom. & \\
\hline I1 & 0,674 & 3,490 & 3,490 & 3,490 & 11,144 & 2,786 & $-0,273$ & $\begin{array}{l}\text { Muy } \\
\text { Adecuado }\end{array}$ \\
\hline $\mathrm{I} 2$ & 0,000 & 3,490 & 3,490 & 3,490 & 10,470 & 2,618 & $-0,104$ & $\begin{array}{l}\text { Muy } \\
\text { Adecuado }\end{array}$ \\
\hline I3 & 0,319 & 3,490 & 3,490 & 3,490 & 10,789 & 2,697 & $-0,184$ & $\begin{array}{l}\text { Muy } \\
\text { Adecuado }\end{array}$ \\
\hline I4 & 0,157 & 1,534 & 3,490 & 3,490 & 8,671 & 2,168 & 0,345 & $\begin{array}{l}\text { Bastante } \\
\text { Adecuado }\end{array}$ \\
\hline I5 & 0,319 & 3,490 & 3,490 & 3,490 & 10,789 & 2,697 & $-0,184$ & $\begin{array}{l}\text { Muy } \\
\text { Adecuado }\end{array}$ \\
\hline I6 & 0,319 & 1,150 & 3,490 & 3,490 & 8,449 & 2,112 & 0,401 & $\begin{array}{l}\text { Bastante } \\
\text { Adecuado }\end{array}$ \\
\hline Suma & 1,788 & 16,644 & 20,940 & 20,940 & 60,312 & 15,078 & & \\
\hline $\begin{array}{l}\text { Promedio } \\
\text { Puntos de } \\
\text { corte }\end{array}$ & 0,298 & 2,774 & 3,490 & 3,490 & $\mathrm{~N}=$ & $\underline{\underline{2,513}}$ & & \\
\hline
\end{tabular}

Fuente: Elaboración propia.

En esta tabla se puede observar que existe consenso entre los expertos en cuanto a que el modelo constituye un aporte novedoso, en sus fundamentos se revelan categorías que contribuyen a comprender y explicar la esencia del proceso de formación del pensamiento reflexivo en estudiantes de las carreras de Ingeniería, las configuraciones del modelo constituyen procesos que en su interrelación posibilitan explicar este proceso formativo, el Sistema de procedimientos didácticos es coherente con el modelo propuesto, sus acciones posibilitan transformaciones importantes en los estudiantes y son factibles de introducir bajo las condiciones actuales del contexto formativo de la Universidad de Ciego de Ávila Máximo Gómez Báez, al ser evaluados todos los aspectos de muy adecuados.

\section{Conclusiones}

El proceso de enseñanza aprendizaje de la Matemática en las carreras de Ingeniería tiene incluido la solución de problemas matemáticos vinculados al perfil profesional, se puede denotar que en este proceso, aún existen dificultades en este sentido, pues no existe la unificación pertinente entre 
la lógica formal de la matemática y la lógica hermenéutica que propicie el desarrollo de un pensamiento reflexivo, llegando a formar profesionales competentes, que asuman los desafíos que se les pueda presentar en su vida como profesionales y como persona, donde prevalezca el amor hacía sí mismo y hacía los demás, aceptando siempre la crítica constructiva como un desafío para potenciar su capacidad creadora en cualquier circunstancia de su vida.

El Sistema de procedimientos didácticos para el proceso de enseñanza aprendizaje de la Matemática en las carreras de Ingeniería, es imprescindible para los estudiantes, pues conlleva a desarrollar el pensamiento reflexivo de los mismos, desde el inicio de la aplicación de sus acciones, se hace desarrollan capacidades en los estudiantes, en su razonamiento, lo cual conlleva a la interiorización y entendimiento de conceptos que resulta difícil de entender por tener el carácter esquemático y poco concreto, mediante la puesta en práctica de los procedimientos adecuados con la fundamentación mínima exigible, así como la sistematización del contenido a través de los vínculos internos entre estos.

El Sistema de procedimientos didácticos tiene acciones que son pertinentes y pueden introducirse fácilmente en el proceso de enseñanza aprendizaje de la Matemática, lo cual se hace manifiesto mediante la puesta en práctica de los procedimientos adecuados aplicados en las carreras de Ingeniería de la Universidad Máximo Gómez Báez, denotándose en los estudiantes nuevas formas reflexivas al resolver problemas matemáticos y al exponer la interpretación de sus resultados enfocados a su perfil profesional, también existe un consenso entre los expertos encuestados hacía la valides de la aplicación del Sistema de procedimientos didácticos en la práctica pedagógica.

\section{Referencias Bibliográficas}

Ackoff, Russell (1986). Planificación de la Empresa del Futuro. Editorial Noriega. México.

Ausubel, David Paul (1968). Educational psychology: a cognitive view. Editorial Holt, Rinehart and Winston. EE. UU.

Bagué, Yamilis Maria; Bravo, Maria de Lourdes; Morales, Yohanna (2021). Una alternativa para lograr la inclusión educativa a través de la resolución de problemas matemáticos. Revista Conrado. Volumen 17, No. 79. Cuba (Pp. 303-309).

Barros, Carlos Alberto (2010). Los Ejemplos en Clase de Matemáticas de Secundaria como Referente del Conocimiento. Tesis de doctorado. Tesis de doctorado en Ciencias. Universidad de Extremadura. España.

Camoli, Américo; Ferreira, María Rosita; Pino, Manuel Guillermo (2020). Resolución de problemas que conduce a ecuaciones diferenciales ordinarias mediante la transformada de Laplace en las carreras universitarias de Angola II CIIE: Aplicación, gestión y uso edificador del conocimiento. Editorial Redipe. EE UU.

Campistrous, Luis. (1992). Lógica y procedimientos lógicos del aprendizaje. Editorial Instituto Central de Ciencias Pedagógicas. Cuba.

Carmona, Nidia Lilis. (2010). El razonamiento en el desarrollo del pensamiento lógico a través de una unidad didáctica basada en el enfoque de resolución de problemas. Tesis de Maestría. Tesis de Maestría en Educación. Universidad Tecnológica de Pereira. Colombia.

Castro, Alexei (2020). La Exploración y modificación de la actitud de los estudiantes de preuniversitario hacia el estudio de las matemáticas. Revista Reduc. Volumen 16, No. 3. Cuba (Pp.465-472).

Davydov, Vasily Visilovich. (1986). Los problemas fundamentales del desarrollo del pensamiento en el proceso de enseñanza. En antología de la psicología pedagogía y de las edades. Editorial Pueblo y Educación. Cuba.

Diéguez, Raquel (2001). Un Modelo del proceso de solución de problemas matemáticos contextualizados en la matemática básica para la carrera de Agronomía. Tesis de Doctorado. Tesis de Doctorado en Ciencias de la Educación Superior. Universidad de Santiago de Cuba. Cuba. 
Duval, Raymound (1993). Registres de représentation sémiotique et functionnement cognitive de la pensée. Annales de Didactique et de Sciences Cognitives. Editorial IREM de Strasbourg. Francia.

Elder, Linda; Paul, Richard (2010). Critical Thinking: Competency Standards Essential for the Cultivation of Intellectual Skills. Revista ERIC. Volumen 34, No. 2. EE UU (Pp. 38-39). https://eric.ed.gov/?id=EJ986272

Ennis, Robert (2013). Cuestionar las evidencias, educar en la reflexión, el estudio del pensamiento crítico y su influjo en la pedagogía del deporte. Revista internacional de ciencias del deporte. Volumen 9, No. 33. Cuba (Pp. 298-30).

Faustino, Arnaldo (2014). La formación del pensamiento matemático-investigativo en los estudiantes de la carrera de licenciatura en matemática. Tesis de Doctorado. Tesis de Doctorado en Ciencias de la Educación Superior. Universidad de Ciego de Ávila "Máximo Gómez Báez". Cuba.

Fernández, C. (2017). Los escalones de la enseñanza - aprendizaje y las fases estratégicas en la solución de problemas de aplicación para la compresión significativa del cálculo infinitesimal. 15 Encuentro Internacional por la Unidad de los Educadores Pedagogía 2017. La Habana, Cuba. https://www.noveduc.com/evento/362

Fuentes, Homero (2009). Pedagogía y Didáctica de la Educación Superior. Editorial Centros de estudios de la Universidad de Oriente. Cuba.

García, Laura (2014). Perfeccionamiento del Proceso de Enseñanza-Aprendizaje de la Matemática en la carrera de Ingeniería Agrícola. Tesis de Maestría. Tesis de Maestría en Ciencias. Universidad Central Martha Abreu. Cuba.

Germán, Yenmy (2017). Aproximación transdisciplinaria en los proyectos de aprendizajes para potenciar el pensamiento crítico en los estudiantes de la uea. 15 Encuentro Internacional por la Unidad de los Educadores Pedagogía 2017. La Habana, Cuba.

Halpern, Diane. (1998). Teaching critical thinking for transfer across domains: Disposition, skills, structure training, and metacognitive monitoring. American Psychologist. Extraido de https://www.semanticscholar.org/paper/Teaching-critical-thinking-for-transfer-across-andHalpern/7d1d8627450c93b5d789b7e164f680639e3961ee

Hernández, Herminia (1995). Nodos cognitivos. Recurso eficiente para el pensamiento matemático. Conferencia Magistral RELME-9. La Habana, Cuba.

Lagos, Jaime Arturo (2017). La enseñanza problémica, un modelo posible en la educación superior. 15 Encuentro Internacional por la Unidad de los Educadores Pedagogía 2017. La Habana, Cuba.

Lenin, Vladimir. (1964). Los presupuestos epistemológicos de la lógica dialéctica. Editora Política Cuadernos Filosóficos. Cuba

Ministerio de Educación Superior (1977). Plan de estudio A, para las carreras de Ingeniería. Cuba

Ministerio de Educación Superior (1986). Plan de estudio B, para las carreras de Ingeniería. Cuba

Ministerio de Educación Superior (1990). Plan de estudio C, para las carreras de Ingeniería. Cuba

Ministerio de Educación Superior (2003). Plan de estudio D, para las carreras de Ingeniería. Cuba

Ministerio de Educación Superior (2018). Plan de estudio E, para las carreras de Ingeniería. Cuba

Moreno, Luis Roberto (2011). Dificultades de aprendizaje en matemática. Editorial XIII CIAEM. Brasil.

Oviedo, Olga Luisa (2015). Una metodología para propiciar el desarrollo de procedimientos lógicos asociados a conceptos en las clases de química de preuniversitario. Editorial Universitaria del Instituto Central de Ciencias Pedagógicas. Cuba.

Paulino, Guzmán David (2019). El razonamiento lógico matemático y su influencia en el rendimiento académico en Matemática I. Tesis de Maestro. Tesis de Maestro en Ciencias 
de la Educación con mención en Educación Matemática. Universidad Enrique Guzmán y Valle. Perú.

Ritacco, Maximiliano (2012). La enseñanza de las matemáticas en contextos de riesgo de exclusión social. Revista de Didáctica de las matemáticas. Volumen 79, No. 2. España (Pp.17-46). http://www.researchgate.net

Lienkov, Edwald Vasilievich (1977). Lógica dialéctica. Colección Socialismo y Libertad. Cuba.

Ruiz, Ramón (2006). Historia y evolución del pensamiento científico. Disponible en:http://www.slideshare.net/GestioPolis.com/historia-y-evolucion-del-pensamientocientifico. Consulta: 20/11/2020.

Torrecilla, Raudel. (2015). La formación de la capacidad modeladora matemática en el ingeniero. Tesis de Doctorado. Tesis de Doctorado en Ciencias de la Educación Superior. Universidad de Ciego de Ávila Máximo Gómez Báez. Cuba.

Wongo, Eurico (2014). Dinámica del proceso de formación interpretativa en la matemática superior. Tesis de Doctorado. Tesis de Doctorado en Ciencias de la Educación Superior. Universidad de Ciego de Ávila Máximo Gómez Báez. Cuba. 